\title{
Changes in local capillarity of pure and hybrid MyHC muscle fiber types after nerve injury in rat extensor digitorum longus muscle (EDL)
}

\author{
Vita Čebašek ${ }^{1}$ and Samo Ribarič ${ }^{2}$ \\ ${ }^{1}$ Institute of Anatomy, University of Ljubljana, Faculty of Medicine, Korytkova 2, Ljubljana, Slovenia \\ ${ }^{2}$ Institute of Pathophysiology University of Ljubljana, Faculty of Medicine, Zaloška cesta 4, Ljubljana, Slovenia \\ ${ }^{1}$ E-Mail vita.cebasek@mf.uni-lj.si ${ }^{1}$ Tel: +386 $15437309^{1}$ \\ ${ }^{2}$ E-Mail samo.ribaric@mf.uni-lj.si ${ }^{2}$
}

\begin{abstract}
Recently, we evaluated capillary indices without discrimination by fiber type in rat extensor digitorum longus muscle (EDL) 4 weeks after nerve cut (NC), after double nerve crush (double NCR) and in two controls, from the start (CON-1) and the end (CON2) of the experiment. In the present study we determined the capillary indices related to specific myosin heavy chain (MyHC) fiber types. Fiber type composition and local capillarity were assessed from a single, composite, multicolor image, where different MyHC fiber types and capillaries were shown simultaneously.

Applying local capillary indices (the number of capillaries around fiber (CAF) and the CAF scaled to fiber perimeter (CAF/FP)), to specific MyHC fiber types, we found changes relevant to neuro-muscular studies. In the NC group, only type- $2 x$ fibers had a significantly lower CAF and in the double NCR group, only type-2a fibers had a higher CAF in comparison to both controls. Both types of nerve injury elicited two responses: a coupled regulation of fiber size and capillarity in the oxidative, type 2a fibers, and a capillarity independent regulation of fiber size in the glycolytic type-2b fibers. All subtypes of type-2 fibers had a better capillary supply (higher CAF/FP) in the NC and double NCR than in CON-2. The highest improvement was observed in type-2b fibers; this change was mirrored in an oxidative shift only in the double NCR group. Adopting fiber type specific capillary indices improves data analysis of rat EDL muscle samples.
\end{abstract}

Keywords: capillary supply, myosin heavy chain (MyHC) muscle fiber type, fluorescence imaging, denervation, re-innervation, morphometry

\section{Introduction}

For muscle fibers types, the precise regulation of capillary supply is important for optimal function, since they exhibit a high specialization and plasticity (Pette and Staron 1988; Schiaffino and Reggiani 1996, 2011; Caiozzo 2002; Schiaffino et al. 2013; Blaauw et al. 2013). Although muscle fibers' diversity is reflected at the structural and biochemical levels, their morphological 
classification is usually made according to the type of their myosin heavy chain (MyHC) protein molecule (Schiaffino and Reggiani 2011). Four different types (isoforms) of MyHC molecule exist in the limb muscles of adult rats (MyHC beta/slow or MyHC type$1<$ MyHC type-2a < MyHC type-2x < MyHC type -2b, listed from the slowest to fastest) (Schiaffino and Reggiani 1994). With a differential distribution they create a continuum of MyHC fiber types, where muscle fibers are usually classified into four pure (expressing (predominately) only one MyHC isoform) and a number of intermediate hybrid types (co-expressing a different MyHC isoforms in varying proportions). Muscle fibers are capable of responding to altered functional demands by changing their phenotype profile (Staron and Pette 1993; Caiozzo 2002; Caiozzo et al. 2003; Schiaffino and Reggiani 2011). The functional relevance of a muscle fiber type is proportional to its relative abundance in the muscle, thus the quantitative assessment of the MyHC muscle fiber types relative distribution reveals the muscle's functional characteristic and also offers an insight into muscle plasticity. An adequate capillary supply of muscle fibers is essential for normal muscle function; a reduced microvascular supply contributes to a reduced functional capacity (Kissane et al. 2018) as evidenced in post-denervation muscle atrophy (Borisov et al. 2000). Additional morphometrical assessments of capillary density during phenotypic transformation of skeletal muscle, may identify new potential drivers of remodeling that could be applicable for development of countermeasures to prevent muscle mass loss (Kissane et al. 2018), not only at denervation, but also in the other conditions associated with denervation (Liu et al. 2016; Piasecki et al. 2016). Although peripheral nerve injury is an effective experimental model for studying plasticity of muscle tissue (Eisenberg and Hood 1994; Midrio 2006; Sun et al. 2006; Wu et al. 2014), these studies rarely included quantitative capillary analysis. However, denervation-induced capillary rarefaction was observed in human (Carpenter and Karpati 1982; Carry et al. 1986) and animal muscles (Tyml et al. 1999; Borisov et al. 2000; Dedkov et al. 2002; Wagatsuma et al. 2005; Ichinose et al. 2008; Čebašek and Ribarič 2016; Paudyal et al. 2018). Capillary rarefaction lags behind denervation-induced atrophy, and capillary loss occurs only after muscle atrophy is almost complete (Paudyal et al. 2018). The start of capillary rarefaction during denervation is muscle specific (Tyml et al. 1999; Cebasek et al. 2006; Paudyal et al. 2018), and progresses with the duration of muscle denervation (Borisov et al. 2000). Besides our own study of the capillary supply of metabolically different muscle fibers types following peripheral nerve injury (Janacek et al. 2009), we found only two more studies; one where the quantitative analysis of capillaries was made in rat anterior tibialis muscle 6-8 weeks after crush of the lateral popliteal nerve, and lower capillary/fiber index than in intact muscles was found for more glycolytic fiber types (Large and Tyler 1985), and the other, where qualitative electron microscopy in long term denervated rat EDL muscle showed that the slow oxidative fibers retained their capillaries for longer than the fast glycolytic fiber types (Borisov et al. 2000). Just recently, it was shown in rat muscles after spinal cord injury (SCI), that changes in muscle capillary supply could be better followed by using geometrical local capillary supply indices in relation to cross sectional area (CSA) and to specific MyHC fiber type (Kissane et al. 2018). However, remodeling of capillaries related to different MyHC types after peripheral nerve injury is largely unexplored, although it was proposed that skeletal muscle capillarity is probably not regulated on a tissue or organ level, but rather on the level of the individual muscle cells (Egginton and Gaffney 2010). 
In a previous study (Čebašek and Ribarič 2016), we reported that capillarity of muscle fibers changed in rat EDL muscle, 4 weeks after two types of sciatic nerve injuries (nerve cut (NC) and double nerve crush (double NCR)). This previous study did not relate capillarity parameters to specific muscle fiber phenotype. The objective of this study was to determine (in the same muscle samples), whether the previously observed changes of capillarity were MyHC muscle fiber type specific. We hypothesized, that differential changes of muscle fibers capillary supply, after nerve denervation, will be accompanied by consistent transformations of MyHC muscle fiber types.

\section{Materials and methods}

Animals and experimental protocol

Sixteen male Wistar rats, of initial body weight from 193g - 233g, were randomly divided into four groups (4 animals per group): (CON-1) - control group 1, where the left EDL was isolated on day 0; (CON-2) - control group 2, where the left EDL was isolated on day 42; (NC)- permanent denervation group, where the left sciatic nerve was cut on day 14 and the left EDL was isolated after 4 weeks, on day 42 and (Double NCR)) - transient denervation group, where the first crush of the left sciatic nerve was done on day 0 , the second crush on day 14, and the left EDL was isolated on day 42 . The experimental protocol is summarised in Table 1. The protocol was approved by the Veterinary Administration of the Ministry for Agriculture, Forestry and Food, Republic of Slovenia (Permit 34401-19/2009/10). Details of the procedure were explained in our previous study (Čebašek and Ribarič 2016).

Tissue cross sections and staining

EDL muscle samples were embedded in an optimal cutting embedding medium (Tissue-Tek: Sakura) at $-22^{\circ} \mathrm{C}$, and $5 \mu \mathrm{m}$ and $10 \mu \mathrm{m}$ transverse tissue slices were cut in a cryostat microtome Leica CM1850 (Leica Microsystems, Germany). To assure equal staining conditions, the samples of all experimental groups were always mounted together on the same microscope slides (Superfrost Plus; Fisher Scientific, Pittsburgh, PA), and stored at $-80^{\circ} \mathrm{C}$ until assayed.

\section{Immuno-fluorescent staining of MyHC-isoforms and lectin-fluorescent staining of capillaries}

Five $\mu \mathrm{m}$ thin tissue sections were stained for morphometrical purposes as previously reported (Ribarič and Čebašek 2013). Briefly, after fixation (for $2 \mathrm{~min}$ in cold acetone) and rinsing (for $20 \mathrm{~min}$ in phosphate-buffered saline (PBS) (0.01 M, pH 7.4) fluoresceinlabelled Griffonia (Bandeira) simplicifolia lectin GSL I (1: 300; FL 1101 Vector Laboratories) and rhodamine-labelled Griffonia (Bandeira) simplicifolia lectin GSL I (1:100); RL 1102 Vector Laboratories) were applied on a tissue section for $2 \mathrm{~h}$ at $4{ }^{\circ} \mathrm{C}$ for visualization of capillaries. After washing in PBS, the cocktail of three primary mouse monoclonal antibodies (directed against three different MyHC isoforms) was applied for $1 \mathrm{~h}$ at room temperature. The following antibodies were used: BA-D5 (of subclass IgG2b, directed against $\beta$ /slow MyHC type-1 (1:100), SC-71 (of subclass IgG1, directed against MyHC type-2a (1:100) and BF-F3 (of class IgM, directed against MyHC type-2b (1:20) (Schiaffino et al. 1989), all purchased from Deutsche Sammlung von Mikroorganismen 
und Zellkulturen (DSMZ, Braunschweig, Germany). Negative control tissue section was processed in the same way, except that the cocktail of primary antibodies was omitted. A series of single stained tissue sections, where MyHC isoforms were visualized with immunoperoxidase staining, were used as positive controls. After washing in PBS, the cocktail of three secondary antibodies (polyclonal goat anti-mouse, subclass specific) was applied for $30 \mathrm{~min}$ at room temperature: Alexa Fluor 350 goat anti-mouse IgG2b - A21140 (1: 350), Alexa Fluor 546 goat anti-mouse IgG1 - A21123 (1: 350) and Alexa Fluor 488 goat anti-mouse IgM - A21042 (1: 350), all purchased from Invitrogen, Carlsbad, Calif., USA. All incubations were performed in dark, after a final rinsing in PBS, the tissue sections were mounted in Elvanol.

\section{Immuno-peroxidase staining of MyHC-isoforms}

The series of $10 \mu \mathrm{m}$ thick transverse tissue sections (successive to $5 \mu \mathrm{m}$ thick tissue section) were used to evaluate muscle fiber types classification that was performed on a multiple stained single tissue section. Successive tissue sections were stained by

"classical indirect immunoperoxidase method" as described previously (Schiaffino et al. 1989). Shortly, each of the primary antibodies, (immunoreactive with a different MyHC isoform) was applied on a separate tissue section for $1 \mathrm{~h}$ at room temperature. The following primary antibodies were used: BA-D5 (directed against $\beta /$ slow MyHC type-1 isoform) (1: 100), SC71 (directed against MyHC type-2a isoform) (1: 100), BF-F3 (directed against MyHC type-2b isoform) (1: 20), and BF-35 (directed against all MyHC isoforms except type-2x) (undiluted); all purchased from Deutsche Sammlung von Mikroorganismen und Zellkulturen (DSMZ, Braunschweig, Germany) were visualized by the same secondary polyclonal rabbit anti-mouse antibodies, peroxidase conjugated (P260; DAKO, Denmark). Primary monoclonal antibody 6H1 antibody (directed against MyHC type-2x isoform) (1:20) (Lucas et al. 2000), purchased from Developmental Studies Hybridoma Bank (DSHB, University of Iowa), was visualized by Novolink polymer detection system as recommended by manufacturer (Novocastra secondary antibodies, Leica Microsystems). Sections incubated without primary antibodies showed no immunoreactivity, confirming the specificity of immunostaining.

\section{Enzyme-histochemical staining and basic histology}

$10 \mu \mathrm{m}$ thick transverse sections (serial to those used for immunohistochemical determination of MyHC muscle fiber types) were stained either histochemically to show changes in the enzymatic activity of muscle cells (succinate dehydrogenase (SDH) (Reichmann and Pette 1984), NADH tetrazolium reductase (TR) (Novikoff et al. 1961) to show presence of oxidative enzymes and the $\alpha$-glycerophosphate dehydrogenase ( $\alpha$-GPDH) (Kugler 1991) to show presence of glycolytic enzymes) or by Haematoxylin and Eosin (HE) (Vacca 1985) to demonstrate changes in basic histology.

Image Acquisition and Morphometrical evaluation

Morphometrical evaluations of capillaries and muscle fibers types were performed on merged images of dark (red, green and blue channels) and white light view, obtained with a Nikon Eclipse E800 microscope (Nikon Corporation, Tokyo, Japan) connected to a digital camera DXM1200F (Nikon Corporation, Tokyo, Japan) at x 20 magnification (objective Plan-Fluor Ph1). Fluorescence was 
excited with a super-high-pressure mercury lamp, and the following Nikon optical filter blocks configuration was used for single channel acquisition in dark light: G-2A (the green excitation optical filter combination), B-2A (the blue excitation filter combination) and UV-2A (the ultraviolet excitation filter combination). Dark and white light views (obtained from exactly the same field of view) were merged with a purpose to achieve better visualization of muscle fiber's outlines, since the basal lamina of muscle fibers was left unstained (Ribarič and Čebašek 2013). White light views (obtained from the series of differently stained successive tissue sections) were used either for detection of MyHC isoforms (visualized by indirect immunoperoxidase method) or for visualization the activity of the main metabolic enzymes or observing the general histological features of muscle tissue.

An image acquisition software Nikon Lucia, version 4.5 (Prague, Czech Republic) was used for acquisition and storage of digital images. All morphometrical measurements and counting were done on merged images obtained from multiple-stained single tissue cross-section, with an area $680 \mu \mathrm{m}$ x $283 \mu \mathrm{m}$ (x-y dimension were 1632 x 680 pixels). Adjustments (if needed) were applied to the entire images in the Adobe Photoshop program (version 7.0; USA) before transferring them into the modular program for interactive image processing Ellipse (ViDiTo, Kosice, Slovakia), where morphometrical measurements were performed. Muscle fiber perimeter (FP; in $\mu \mathrm{m}$ ) and number of capillaries around fiber (CAF) were assessed for 4118 fibers of different MyHC types (Table 2) from two random non-overlapping fields ( $257 \pm 94$ muscle fibers per muscle sample). Local indices of capillary supply estimated as CAF per fiber perimeter $\left(\mathrm{CAF} / \mathrm{FP}\right.$, in $\left.\mu \mathrm{m}^{-1} \times 10^{-3}\right)$ were calculated in Microsoft Office Excel program (Ribarič and Čebašek 2013; Čebašek and Ribarič 2016).

\section{Muscle Fiber Type Identification}

The MyHC muscle fiber type determination was performed on multicolor fluorescent images obtained from a single multiple-stained tissue section, where the expression of $\mathrm{MyHC}$ isoforms was revealed by the set of three primary antibodies immunoreactive with MyHC isoforms type-1 (BA-D5), type-2a (SC-71) and type-2b (BF-F3) and the set of three secondary antibodies (isotype specific) conjugated by three different flourochromes (Alexa Fluor 350, - 546 and -488, respectively). The pure and hybrid MyHC muscle fibers types have been identified according to the specific color shade (Fig. 1). Prior to the analysis of each muscle group, the identification of muscle fiber type, made on a single multicolor fluorescence image (RGB image), was checked on the series of classically stained tissue sections, where each of the MyHC isoform type was visualized separately (by immunoperoxidase reaction) on separate (successive) tissue slices (Figs. 2-5).

Some of the hybrid MyHC muscle fibers types that expressed more than two different MyHC isoforms in unequal proportions, and the hybrid muscle fibers types that co-express type-1 and type-2x MyHC isoforms could not be reliably identified without an additional checking on classically stained tissue cross section, where the expression of type-2x MyHC isoform was shown. Therefore some of the hybrid fiber types were probably misclassified and included in one of the "nearby" fiber types from the muscle fiber type's continuum (Caiozzo et al. 2003). 
Statistics

Morphological data are presented as mean \pm standard deviation (SD). Differences in mean values were evaluated by a one-way ANOVA, applying the Bonferroni correction. Results were considered to be statistically significant when $\mathrm{P}<0.05$. Statistical analyses were performed with IBM SPSS Statistics Version 25.

\section{Results}

Wistar rats were subjected to a permanent denervation (nerve cut (NC)) or a transient denervation (double nerve crush (double NCR)), and four weeks later the Extensor digitorum longus muscle (EDL) was examined. As previously reported (Čebašek and Ribarič, 2016), a severe atrophy of denervated EDL muscle (NC group) was found in comparison to both controls (CON-1 and CON-2, age/weight-matched control from the start and the end of experiment) and to re-innervated group (double NCR). The global $(\mathrm{C} / \mathrm{F})$ and the general local (CAF, Lcap) indices of capillarization were lower in denervated than in re-innervated group. In this study, we focused on the local capillarization of different pure and hybrid MyHC-fiber types.

\section{Muscle Fiber Type Composition}

The numerical and areal proportions of the individual pure (expressing predominately only one MyHC isoform) and of pooled hybrid MyHC muscle fibers types (co-expressing a different MyHC isoforms in varying proportions) are presented in Fig. 6. The total number of experimental muscle fibers is reported in Table 2.

\section{Denervation versus control}

Permanent denervation (NC) did not induce any significant differences in numerical percentages (frequency) of muscle fiber types. The areal percentage of the type-2a fibers increased and was about 2-times higher than in CON-1 and CON-2 groups (both p<0.05); the areal percentage of type-2b fibers decreased and was about $35 \%$ lower than in CON-1 and CON-2 (both $p<0.01)$ ). The effect of these changes was that the areal proportions among different type-2 pure fibers became almost equal.

\section{Re-innervation versus control and denervation}

Transient denervation (double NCR) induced changes in numerical percentages (frequency) of different type-2 muscle fiber subtypes: the frequency of type-2b fibers decreased and was about 10-times lower than in CON-1, CON-2 and denervated (NC) group (all $\mathrm{p}<0.001$ ); the frequency of pooled hybrid fibers types increased and was about 2-times higher than in $\mathrm{CON}-1, \mathrm{CON}-2$ (both $\mathrm{p}<0.01)$ and denervated $(\mathrm{NC})$ group $(\mathrm{p}<0.05)$. Additionally, the areal percentage of type-2x fibers increased in re-innervated group (double NCR) and was about 2-times higher than in all other groups $(\mathrm{p}<0.01)$ (Fig. 6). Histochemical staining for visualization of enzymes involved in the metabolic pathways of muscle cells confirmed these results; all of the re-innervated fibers showed a higher staining intensity for oxidative enzymes (Fig. 7). 
Muscle Fiber Type Size

Both types of nerve injuries affected only the size of type-2 phenotypes. The permanent denervation (NC) decreased and the transient denervation (double NCR) increased their size, but the degree of the size changes differs among different type- 2 fibers subtypes (the difference was the biggest between the slowest (type-2a) and the fastest (type-2b) muscle fibers). Although atrophy of the type$2 \mathrm{~b}$ muscle fibers was among the biggest after permanent denervation (NC), the increase of their size was the smallest after the transient denervation. The changes of muscle fiber types size, and the statistical differences within and between groups are given in Table 3. Measurements of each muscle fiber type were performed from multicolor fluorescent images, where the different muscle fiber types were shown simultaneously (Figs. 1-5).

\section{Denervation versus control}

Permanent denervation (NC) did not induce any significant changes in the size of type-1 muscle fibers. Different subtypes of type2 fibers decreased their size diversely. In comparison to CON-1 group, atrophy of type-2 fibers subtypes was from 10\% to $37 \%$; (for type-2a $(-10 \%)(\mathrm{p}<0.05)$, for type-2xb $(-22 \%)(\mathrm{p}<0.05)$, for type-2x $(-25 \%)(\mathrm{p}<0.001)$ and for type-2b $(-37 \%)(\mathrm{p}<0.01))$. In comparison to CON-2 group, atrophy was higher, 25\% to 50\%, (for type-2a (-25\%) (p<0.01), for type-2ax (-30\%), for type-2xb ($38 \%)$, for type-2x $(-40 \%)$ and for type-2b $(-50 \%)($ all $\mathrm{p}<0.001)$

\section{Re-innervation versus control and denervation}

Transient denervation (Double NCR) induced an increase of the size of type-2 fibers, but different subtypes changed their size diversely: the size of the three slowest type-2 fibers became larger compared to the initial size in CON-1 group (for type-2a $21 \%$ and for type-2x $20 \%$ (both $\mathrm{p}<0.01$ ), and for type-2ax $18 \%(\mathrm{p}<0.001)$ ). In comparison to CON-2 group, only the size of the fastest type-2b muscle fibers remained smaller $(-24 \%, \mathrm{p}<0.001)$. In comparison to the denervated group, all re-innervated type- 2 fibers were larger by $34 \%$ to $58 \%$ : type-2ax $(+34 \%)$, type- $2 \mathrm{a}(+36 \%)$, type- $2 \mathrm{xb}(+44 \%)($ all $\mathrm{p}<0.001)$, type- $2 \mathrm{~b}(+50 \%)(\mathrm{p}<0.01)$ and type$2 x$ fibers $(+58 \%)(\mathrm{p}<0.001)$.

\section{Muscle Fiber Type Capillarization}

Neither of the sciatic nerve injuries changed the capillarization of muscle fiber types 1 but only of type-2 muscle fibers subtypes. The two injuries had opposite effects on the capillary growth: the permanent denervation (NC) decreased and the transient denervation (double NCR) increased the growth of capillaries, the degree of changes differed among different type-2 subtypes. Comparison of local capillarity for all pure types $(-1,-2 \mathrm{a},-2 \mathrm{x},-2 \mathrm{~b})$ and the two most frequent hybrid types (2ax and $2 \mathrm{xb})$ are graphically presented (Fig. 8). Values are means \pm SD.

\section{Denervation versus control}

Permanent denervation (NC) induced capillary loss only in type-2x fibers, with a lower CAF compared to CON-1 (- 31\%, $<<0.01)$ and to CON-2 $(-20 \%, \mathrm{p}<0.05)$. However, an increased CAF/FP ratio compared to CON-2 was found in the three fastest type-2 
fibers subtypes (type-2x $(+35 \%, \mathrm{p}<0.01)$, type- $2 \mathrm{xb}(+59 \%, \mathrm{p}<0.05)$ and type-2b $(+65 \%, \mathrm{p}<0.05))$, where the rate of atrophy exceeded the capillary rarefaction.

\section{Re-innervation versus control and denervation}

Transient denervation (double NCR) induced a large capillary proliferation, but a higher CAF, compared with both controls was only around type-2a fibers ( $+26 \%$ vs. $\mathrm{CON}-1$ and $+36 \%$ vs.CON-2, both $\mathrm{p}<0.01)$. Compared with CON-2, a higher CAF was found in the three additional type-2 phenotypes; for type-2x $(+30 \%, \mathrm{p}<0.01)$, type- $2 \mathrm{~b}(+39 \%, \mathrm{p}<0.05)$ and for type-2xb $(+52 \%, \mathrm{p}<0.05)$. In comparison to the denervated group, all re-innervated pure type- 2 fibers and the two hybrid muscle fibers types had a higher CAF; type-2a $(+55 \%, \mathrm{p}<0.001)$, type-2b $(+57 \%, \mathrm{p}<0.01)$, type-2x $(+67 \%, \mathrm{p}<0.001)$, type-2ax $(+43 \%, \mathrm{p}<0.05)$, and type-2xb $(+55 \%$, p $<0.05)$. Compared only with $\mathrm{CON}-2$, the highest CAF/FP was found across the several type-2 phenotypes: type-2ax $(+31 \%$, $\mathrm{p}<0.05)$, type-2a $(+36 \%, \mathrm{p}<0.01)$, type-2x $(+39 \%, \mathrm{p}<0.01)$, type-2xb $(+68 \%, \mathrm{p}<0.01)$ and type-2b $(+88 \%, \mathrm{p}<0.01)$. A higher $\mathrm{CAF} / \mathrm{FP}$ in muscle fibers where the fiber size is similar in both groups (i.e. the fiber type-2a and type-2ax) indicates that a better capillary supply (estimated by the CAF/FP ratio) was achieved as a net effect of capillary proliferation.

\section{Discussion}

\section{Denervation}

Four weeks after permanent denervation (after NC), we observed capillary rarefaction across several fiber types, but only the $M y H C$ type- $2 x$ a group had a significantly decreased CAF value $(-30 \%$ vs. CON-1 (p<0.01) and $-20 \%$ vs. CON-2 (p<0.05)) (Fig. 8). These denervation results are in agreement with the results of our previous study (Čebašek and Ribarič 2016): (a) a significant capillary loss $(-25 \%$ vs. CON-1 ( $<<0.05))$ was detected by using the general, MyHC fiber type unspecific, local index of capillarity CAF; (b) the global capillary index $(\mathrm{C} / \mathrm{F})$, a measure of capillary rarefaction and estimated as capillary number per muscle fiber number, was not significantly different compared to controls (-23\% vs. CON-1 and $-11 \%$ vs. CON-2) and (c) the higher number of capillaries per $\mathrm{mm}^{2}(\mathrm{CD}$ index $)$ vs. CON-2 $(+50 \%, \mathrm{p}<0.001)$, only reflected severe atrophy of muscle fibers after permanent denervation. Results of the present study showed a better capillary supply (higher CAF/FP compared with CON-2) in the three fastest type-2 phenotypes (type-2x $(+35 \%, \mathrm{p}<0.01)$, type-2xb $(+59 \%, \mathrm{p}<0.05)$ and type- $2 \mathrm{~b}(+65 \%, \mathrm{p}<0.05)$, that are in the intact EDL also the largest (Table 3 and Fig. 8). These comparisons of results clearly show, how the use of specific local capillarity indices (related to the individual MyHC muscle fiber type) minimize scale dependency (i.e. the muscle fiber's size and phenotype), and could better detect small local variations in capillary supply, at least in rat EDL muscle, where the distinct MyHC muscle fiber types also differ in size. Therefore, we suggest that in studies, where only small changes of anatomical capillarity are expected, specific local parameters of capillarity are more sensitive and could reveal early changes in capillarity.

To our knowledge, this is the first report where capillary supply after peripheral nerve injury was studied in relation to specific MyHC fiber type. Only one additional study, where efficacy of different therapeutic interventions after injury to the central nerve system (spinal cord injury - SCI) were followed, used indices of local capillarity, related to MyHC type-1, $-2 \mathrm{a}$ and $-2 \mathrm{~b}$ (Kissane et 
al. 2018). Both types of injuries (central and peripheral) induced muscle atrophy, however, after SCI, in target skeletal muscle, oxidative fibers typically atrophied prior to glycolytic fibers (Durozard et al. 2000) and after peripheral nerve injury the atrophy of slow (oxidative) fibers is more pronounced only in slow-twitch muscles and not in fast muscles (as is EDL) (Blaauw et al. 2013). Thus, we cannot directly compare our results to any other similar study, and can only speculate about the underlying mechanisms of our specific finding, the capillary rarefaction around muscle fibers type- $2 x$. This selective effect of denervation found in rat EDL, 4 weeks after nerve cut, is consistent with our previous study, where we found SDH-negative type-2x fibers in rat EDL, 2 weeks after nerve cut (Cebasek et al. 2007). Why type-2x fibers are the most sensitive to denervation-induced loss of mitochondria (reported in our previous study) and loss of capillaries (reported in the present study), we do not know. It seems that mitochondria and capillaries are affected in tandem. In studies, where molecular mechanisms responsible for denervation-induced atrophy were investigated, a markedly higher production of reactive oxygen species (ROS) was found in subsarcolemmal (SS) mitochondria (Adhihetty et al. 2007). Since type-2x fibers have a high density of SS mitochondria, we speculate that a higher ROS production from SS mitochondria following denervation might cause the observed higher susceptibility of capillaries around type-2x fibers (and concomitant regression of adjacent capillaries).

A loss of fiber type differentiation visible after staining for succinate dehydrogenase (SDH) (Lexell et al. 1985), and a reduced SDH subsarcolemmal density after denervation (Tomanek and Lund 1973), have been observed by previous studies. Type-2x fibers had an intrinsically higher capillary supply $(\mathrm{CAF} / \mathrm{FP})$ than the type-2b fibers (in CON-2 group values were $2.54 \pm 0.19$ v.s. $1.70 \pm 0.06$ $(\mathrm{p}<0.05))$; a higher susceptibility of type-2x fibers seems to be in contradiction with the speculation by Borisov and co-workers (2000) that muscle fibers with a higher capillary supply are less sensitive to denervation. However, if we consider the decrease in fiber size separately, without capillary loss, the degree of their atrophy is in-between of the other two type- 2 pure fibers $(2 a<2 x<2 b)$, and this is in accordance with their postulation (Table 3; the differences (in percentages) of FP between denervated and CON-1 were the following: for type-2a $-10 \%(\mathrm{p}<0.05)<$ type- $2 \mathrm{x}-25 \%(\mathrm{p}<0.001)<$ type- $2 \mathrm{~b}-37 \%(\mathrm{p}<0.01)$ and between denervated and CON-2: for type-2a $-25 \%(\mathrm{p}<0.01)<$ type- $2 \mathrm{x}-40 \%(\mathrm{p}<0.001)<$ type $-2 \mathrm{~b}-50 \%(\mathrm{p}<0.001))$.

Although selective capillary rarefaction found around type-2x fibers could be muscle specific, and the consequence of some specific circumstances that exist only in rat EDL muscle, our finding might be still applicable to quantitative analysis of local capillarity in other muscles. Namely, it may shorten the time for morphometrical analysis, if screening for capillary rarefaction would be started around type-2x fibers (if muscle of similar fiber type composition is studied) or around fibers that are in a particular skeletal muscle the largest among oxidative fibers and have the highest basal CAF value (the property of the type-2x fibers).

It might be expected that higher CAF/FP values, found in denervated type-2 muscle fibers subtypes, indicate also a higher oxidative metabolism of these fibers, because it was shown that a higher Lcap/Sfib ratio (ratio between capillary length and muscle fiber surface) is characteristic for muscle fibers with a higher rate of oxidative metabolism (if muscle fibers of similar size are compared) (Cebasek 2005). However, staining for succinic dehydrogenase (SDH) reveals a rather lower histochemical activity of oxidative enzyme in denervated muscle fibers than in control, clearly visible also without quantification and without densitometry (Fig. 7). 
Additionally, the contrast of the enzymatic profiles between muscle fibers types tended to disappear in denervated muscle; muscle fibers with previously the highest rates of histochemical activity lost the most of their activity. Similar changes in enzymatic profile of denervated muscle cells were reported either previously, in histochemical or biochemical studies (Nachmias and Padykula 1958; Hogan et al. 1965; Romanul and Hogan 1965) or in recent proteomic studies, where changes in gene expression of several oxidative and glycolytic enzymes (proteins) were detected (Otis et al. 2004; Lang et al. 2018). However, in our present study, a higher CAF/FP of the three fastest denervated type-2 subtypes was not enough to trigger the switching of muscle MyHC fiber phenotype, since we did not find any changes in numerical proportions of MyHC fiber types between denervated and control EDL (Fig. 6).

Consistent with a general "fast to slow" paradigm described elsewhere (Schiaffino et al. 2013) the area proportion of type-2b fibers decreased from $51.72 \pm 5.23 \%$ in CON-1, and $52.28 \pm 6.68$ in CON-2, to $33.45 \pm 2.92$ in denervated muscles (both p <0.01) (Fig. 6). Traditionally, the "fast to slow" shift in fast-twitch denervated muscles was estimated by different approaches: 1) in histochemical studies (on the cell level) it was shown by preferential atrophy of fast type-2 muscle fibers (Tomanek and Lund 1973; Jaweed et al. 1975; Carpenter and Karpati 1982); 2) in biochemical studies (on the protein level) a lower expression of fast MyHC isoforms was measured by analysis of pooled myofibrils (Jakubiec-Puka et al. 1999); and 3) in microscopic studies (on the ultrastructural level) a decreased proportion of FG fibers and an increased proportion of hybrid FOG/FG or FOG/SO fibers was detected by electronic microscopy (Jakubiec-Puka et al. 1990).

In contrast to our present study, (where we did not find any changes in frequency), significant changes in numerical proportions of MyHC muscle fibers types were described in two studies, where a lower proportion of type-2x and a higher proportion of type-2ax (Cebasek et al. 2007) and a lower proportion of type-2b and a higher proportion of hybrid type-Emb/2a and type-Emb/2a/2x fibers were found in 2 weeks denervated rat EDL (Michel et al. 1996). These results cannot be compared with ours, because of different methodology, protocols and controls.

We speculate that the highest atrophy of the largest fibers, the most rapidly loss of histochemical activities of enzymes in those fibers, where activity of these particular enzymes are basically the highest, are part of a highly coordinated response that is regulated either on tissue or organ level, to make unused denervated muscle tissue more uniform and energetically favorable. On the other hand, we agree with the statement of Egginton and Gaffney (2010) that the placement of each individual capillary should be regulated on the scale of individual cells. Thus, we believe that a selective loss of capillaries found around denervated type-2x could be the effect of some strong mismatch between capillary supply and demand that occurs specifically around them.

\section{Re-innervation}

Four weeks after transient denervation (after double-NCR) a capillary proliferation (a higher CAF) was observed: (a) only around muscle fibers MyHC type-2a, when compared to CON-1 (+26\%, p<0.01); (b) around four MyHC fiber types, when compared to CON-2 (type-2x $(+30 \%, \mathrm{p}<0.01)$, type-2a $(+36 \%, \mathrm{p}<0.01)$, type-2b $(+39 \%, \mathrm{p}<0.05)$ and type-2xb $(+52 \%, \mathrm{p}<0.05))$; and $(\mathrm{c})$ around all MyHC type-2 phenotypes, when compared to the permanent denervated group (after NC); the least in type-2ax (+43\%, $<<0.05)$ 
and the most in type-2x $(+67 \%, \mathrm{p}<0.001)($ Fig. 8). These results are in agreement with our previous study (Čebašek and Ribarič 2016), where: (a) a higher capillarity than in all other groups (+20\% vs. CON-1 (p<0.05), $+37 \%$ vs. CON-2 (p<0.001), and $+61 \%$ vs. NC ( $\mathrm{p}<0.001)$ ) was found by using the general local index of capillarity CAF; (b) by using the global capillary index $(\mathrm{C} / \mathrm{F})$, estimated as capillary number per muscle fiber number, a higher capillarity was found only vs. CON-2 $(+51 \%$, p <0.01) and vs. NC $(+70 \%, \mathrm{p}<0.001))$, but not vs. CON-1 (+30\% was not significant); (c) by using the global capillary density, expressed as a number of capillaries per $\mathrm{mm}^{2}$ (CD index), a higher capillarity was found only vs. CON-2 (+117\%, p<0.001). By using the CAF/FP, a better capillary supply was found only vs. CON-2 $(+63 \%, \mathrm{p}<0.001)$. The same samples were re-analysed in the present study, with the MyHC type specific local index CAF/FP. We measured a better capillary supply, compared to controls, only in the $M y H C$ type-2 phenotypes (from $+31 \%$ in type- $2 \mathrm{ax}(\mathrm{p}<0.05)$ to $+88 \%$ in type- $2 \mathrm{~b}(\mathrm{p}<0.01)$ (Fig. 8$)$.

Quantitative analysis of capillaries is rarely applied for analysis of muscle tissue following nerve crush. Beside our previous 3D study of capillaries of metabolically different fibers types in rat EDL following nerve injury, where the assessment of fiber types was made according to histochemical activity of NADH (Janacek et al. 2009), we found only one study in the literature, where quantitative analysis of capillaries following nerve crush was made in relation to several different muscle fiber types (Large and Tyler 1985). In contrast to our present study, where we found a specific capillary proliferation around re-innervated type- 2 fibers, in both of the above mentioned previous studies, only a minor alteration in capillary distribution was reported. In our previous study of metabolically different fibers types, we did not find any significant changes (Janacek et al. 2009), and in the study of Large and Tyler (1985), even a lower capillarity of glycolytic fibers types was reported. Such controversial results are most likely due to a different type of nerve injury used in these studies; in the present study we made the double nerve crush of sciatic nerve in a time period of 2 weeks, and in both previous studies, the nerve crush was made only once. We hypothesize, that the first crush could affect the muscle tissue in such a way to become more sensitive for the second crush, as a catalyst, which could elicit in muscle tissue a larger response and plasticity. A similar explanation was given recently in a study of denervation-induced changes in gastrocnemius muscles of adult and old rats, where the authors suggested that the satellite cells, ribosomes and capillary density, in muscle tissue might be even more responsive during the remodeling time, when the muscle is still adapting to denervation (Paudyal et al. 2018).

Since both the EDL muscle from the present study, and the tibialis anterior muscle from the study of Large and Tyler (1985), are fast twitch (mixed) muscles and ankle (dorsal) flexors, (have a similar fiber type composition) we attribute the diverse result to the usage of different experimental protocols.

According to Large and Tyler (1985), their reported lower value of capillarity of glycolytic muscle fibers ( $\alpha \mathrm{W}$ fibers), found in reinnervated muscle, might be a consequence of artefactual high values found in the normal muscle. Namely, they claimed that glycolytic muscle fibers are only rarely surrounded by only glycolytic fibers in a normal muscle, and that neighboring oxidative fibers should have affected the results. We are aware that this "effect of neighboring fibers" could affect also our results, and that a higher CAF value of type-2a fibers (found in re-innervated muscle in comparison to CON-1), might only reflect the fact, that in a 
re-innervated muscle, the type-2a fibers are more frequently surrounded by type-2a fibers than in a normal intact muscle. We suggest an alternative explanation, a higher concentration of neuronal NO synthases (nNOS) in the sarcolemma of type-2a fibers (Planitzer et al. 2001), generating of a higher concentration of nitric oxide (NO). NO diffuses from muscle fiber into the surrounding milieu/tissue, where it could activate signaling cascades for angiogenesis (Ignarro et al. 1999), and/or coordinate the paracrine interaction between skeletal muscle cells and capillaries (Baum et al. 2013). There could be alternative explanations, since a complex orchestration of pro- and antiangiogenic signals, at various levels, is maintaining an adequate capillary supply of muscle fibers (Olfert and Birot 2011; Hoier et al. 2012; Olfert et al. 2016), which is reflected in the anatomical density of capillaries, thus in the CAF.

Increased capillarity of type-2a fibers was also found in mouse plantaris muscle, after 4 weeks of voluntary running, where an enhanced angiogenesis was accompanied by a "fast-to-slow shift" (Waters et al. 2004); this is a similar tissue transformation that was found in our study in rat EDL 4 weeks following nerve crush. In the active phase of muscle adaptation (day 7), they found an increased concentration of vascular endothelial growth factor (VEGF), which was initially associated with an increased capillarity in type- $2 b$ and type-2x fibers. But this increase was only transient, presumably before conversion of type-2b and type-2x fibers into fibers type-2a (since at day 28 , they found an increased percentage of type-2a fibers and an increased capillary contact in type-2a). Their results suggest that an increased capillary density could play a permissive role for phenotypic changes of muscle fiber types. In our present study, all of the re-innervated type-2 fibers had a higher CAF/FP than their counterparts in CON-2, thus a better capillary supply. Contrary to the denervated muscle, where a higher CAF/FP was achieved passively (simply because the regression of capillaries lagged behind the atrophy of muscle fibers), the higher CAF/FP ratio in re-innervated muscle was achieved by active capillary proliferation that (proportionally) overtakes the increase of muscle fibers. A better capillary supply is associated with a higher histochemical activity of oxidative enzyme SDH (qualitative estimation) and with a massive conversion (from glycolytic to oxidative form) of muscle fiber type-2b (quantitative estimation), since a lower numerical percentage of type-2b and a higher percentage of pooled hybrid fibers was found in re-innervated muscle (Fig. 6). The substantial conversion of the glycolytic type-2b fibers into the more oxidative muscle fibers types, is consistent with the fact, that type-2b fiber is the only one, that 4 weeks after double NCR, has not yet achieved the size of their counterparts in CON-2 ( $\mathrm{p}<0.01)$ (Table 3$)$. The slower and more oxidative type2a fibers, (that also had a higher CAF/FP in re-innervated muscle than in the CON-2 after 4 weeks), completely achieved the size of their counterparts in $\mathrm{CON}-2$ and retained their high oxidative potential. Thus, it seems, that the intracellular (hypertrophic) signal in the more oxidative muscle cells is more in balance with the (mitogen) induction in the nearby endothelial cells, since the size and the capillary supply of type-2a fibers change almost proportionally. This finding, valid for type-2a fibers, is in line with a hypothesis, that the size and the capillary supply of a muscle fiber share same/similar control mechanisms (Barnouin et al. 2017). It may be, that the most glycolytic type-2b fibers can regulate their size "more independently" thus separately from the capillary supply, since they can retain an un-proportional small fiber size (in double NCR) despite of the same "luxury of capillary supply" that is available to type-2a fibers. This special ability, of deferential regulation of fiber's size, leads to an improved CAF/FP ratio and probably has 
a permissive role to switch the muscle fiber towards a more oxidative metabolism. Further studies are needed to elucidate if special intrinsic properties of type-2b fibers might be responsible for this effect (some special chemo- or mechanic receptors anchored into the sarcolemma or inside the cell) or if some specific properties of the nearby milieu - extracellular matrix regulate muscle fiber metabolism (Egginton and Ross 1989; Egginton et al. 1998; Egginton and Hudlická 2000; Germinario et al. 2002; Roy et al. 2002; Simon et al. 2003; Lang et al. 2018).

\section{Conclusion}

The multicolor fluorescent technique, for simultaneous visualization of capillaries and MyHC isoforms, is useful for morphometrical analyses, especially around considerably atrophic, or hybrid muscle fibers types. In rat EDL muscle, where distinct fiber types also differ in size, the usage of the local capillary supply indices related to MyHC fiber type, improves data resolution, compared to our previous study, where only the global and the general local indices (related only to fiber size) were used. Although both studies detected capillary rarefaction (after NC) and proliferation (after double NCR), the results of the present study offer an additional information about the specific changes in capillarity and the specific strategies of capillary regulation around the different fiber types. For example, in the NC group, the type-2x muscle fibers (the largest among oxidative fibers) were the most sensitive to capillary rarefication; in the double NCR group, the most pronounced proliferation of capillaries occurred around the type-2a muscle fibers, and the highest improvement in capillary supply, compared with CON-2, was detected in the muscle fibers type-2b. Our results suggest, that following nerve injury, a coupled regulation of fiber size and capillarity exists in the oxidative fiber type (type2a), maintaining oxidative metabolism of muscle cells, and that a more independently regulated fiber size exists in the glycolytic fibers (type-2b), that offers them the flexibility for an easier/faster change of metabolism from a glycolytic to more a oxidative form.

\section{Acknowledgements and Funding Information}

We thank Mrs. Majda Črnak-Maasarani, Mrs. Andreja Vidmar, Mrs. Nataša Pollak, Mr. Marko Slak and Mr. Milan Števanec for their technical assistance. The research was supported by the Slovenian Research Agency (P3-0043 and P3-0171).

\section{Compliance with ethical standards}

Conflict of interest

The authors declare that they have no conflict of interest.

\section{References}

Adhihetty PJ, O'Leary MFN, Chabi B, Wicks KL, Hood Da (2007) Effect of denervation on mitochondrially mediated apoptosis in skeletal muscle. J Appl Physiol (1985) 102 (3):1143-1151. doi:10.1152/japplphysiol.00768.2006

Barnouin Y, McPhee JS, Butler-Browne G, Bosutti A, De Vito G, Jones DA, Narici M, Behin A, Hogrel JY, Degens H (2017) Coupling between skeletal muscle fiber size and capillarization is maintained during healthy aging. J Cachexia Sarcopenia Muscle. doi:10.1002/jcsm.12194

Baum O, Vieregge M, Koch P, Gül S, Hahn S, Huber-Abel FaM, Pries AR, Hoppeler H (2013) Phenotype of capillaries in skeletal muscle of nNOS-knockout mice. Am J Physiol Regul Integr Comp Physiol 304 (12):R1175-1182. doi:10.1152/ajpregu.00434.2012

Blaauw B, Schiaffino S, Reggiani C (2013) Mechanisms modulating skeletal muscle phenotype. Compr Physiol 3 (4):1645-1687. doi:10.1002/cphy.c130009 
Borisov AB, Huang SK, Carlson BM (2000) Remodeling of the vascular bed and progressive loss of capillaries in denervated skeletal muscle. Anat Rec 258 (3):292-304

Caiozzo VJ (2002) Plasticity of skeletal muscle phenotype: mechanical consequences. Muscle Nerve 26 (6):740-768. doi:10.1002/mus.10271

Caiozzo VJ, Baker MJ, Huang K, Chou H, Wu YZ, Baldwin KM (2003) Single-fiber myosin heavy chain polymorphism: how many patterns and what proportions? Am J Physiol Regul Integr Comp Physiol 285 (3):R570-580. doi:10.1152/ajpregu.00646.2002

Carpenter S, Karpati G (1982) Necrosis of capillaries in denervation atrophy of human skeletal muscle. Muscle Nerve 5 (3):250254. doi:10.1002/mus.880050313

Carry MR, Ringel SP, Starcevich JM (1986) Distribution of capillaries in normal and diseased human skeletal muscle. Muscle Nerve 9 (5):445-454. doi:10.1002/mus.880090510

Cebasek V, Erzen I, Vyhnal A, Janácek J, Ribaric S, Kubínová L (2010) The estimation error of skeletal muscle capillary supply is significantly reduced by 3D method. Microvasc Res 79 (1):40-46. doi:10.1016/j.mvr.2009.11.005

Cebasek V, Kubínová L, Janácek J, Ribaric S, Erzen I (2007) Adaptation of muscle fibre types and capillary network to acute denervation and shortlasting re-innervation. Cell Tissue Res 330 (2):279-289. doi:10.1007/s00441-007-0484-5

Cebasek V, Kubinova L, Ribaric S, Erzen I (2005) Capillary network in slow and fast muscles and in oxidative and glycolytic muscle fibres. Image Anal Stereol 24:51-58

Cebasek V, Radochová B, Ribaric S, Kubínová L, Erzen I (2006) Nerve injury affects the capillary supply in rat slow and fast muscles differently. Cell Tissue Res 323 (2):305-312. doi:10.1007/s00441-005-0071-6

Čebašek V, Ribarič S (2016) Changes in the Capillarity of the Rat Extensor Digitorum Longus Muscle 4 Weeks after Nerve Injury Studied by 2D Measurement Methods. Cells Tissues Organs 201 (3):211-219. doi:10.1159/000444140

Dedkov EI, Kostrominova TY, Borisov AB, Carlson BM (2002) Resistance vessel remodeling and reparative angiogenesis in the microcirculatory bed of long-term denervated skeletal muscles. Microvasc Res 63 (1):96-114. doi:10.1006/mvre.2001.2372

Durozard D, Gabrielle C, Baverel G (2000) Metabolism of rat skeletal muscle after spinal cord transection. Muscle Nerve 23 (10):1561-1568

Egginton S, Gaffney E (2010) Tissue capillary supply--it's quality not quantity that counts! Experimental physiology 95 (10):971979. doi:10.1113/expphysiol.2010.053421

Egginton S, Hudlická O (2000) Selective long-term electrical stimulation of fast glycolytic fibres increases capillary supply but not oxidative enzyme activity in rat skeletal muscles. Exp Physiol 85 (5):567-573

Egginton S, Hudlicka O, Brown MD, Walter H, Weiss JB, Bate A (1998) Capillary growth in relation to blood flow and performance in overloaded rat skeletal muscle. J Appl Physiol (1985) 85 (6):2025-2032. doi:10.1152/jappl.1998.85.6.2025

Egginton S, Ross HF (1989) Influence of muscle phenotype on local capillary supply. Adv Exp Med Biol 248:281-291

Eisenberg HA, Hood DA (1994) Blood flow, mitochondria, and performance in skeletal muscle after denervation and re-innervation. J Appl Physiol (1985) 76 (2):859-866. doi:10.1152/jappl.1994.76.2.859

Germinario E, Esposito A, Megighian A, Midrio M, Biral D, Betto R, Danieli-Betto D (2002) Early changes of type 2B fibers after denervation of rat EDL skeletal muscle. J Appl Physiol 92 (5):2045-2052. doi:10.1152/japplphysiol.00673.2001

Hogan EL, Dawson DM, Romanul FC (1965) Enzymatic changes in denervated muscle. II. Biochemical studies. Arch Neurol 13 (3):274-282

Hoier B, Nordsborg N, Andersen S, Jensen L, Nybo L, Bangsbo J, Hellsten Y (2012) Pro- and anti-angiogenic factors in human skeletal muscle in response to acute exercise and training. The Journal of physiology 590 (Pt 3):595-606. doi:10.1113/jphysiol.2011.216135

Ichinose E, Kurose T, Daitoku D, Kawamata S (2008) The skeletal muscle vascular supply closely correlates with the muscle fiber surface area in the rat. Arch Histol Cytol 71 (1):45-57

Ignarro LJ, Cirino G, Casini A, Napoli C (1999) Nitric oxide as a signaling molecule in the vascular system: an overview. Journal of cardiovascular pharmacology 34 (6):879-886

Jakubiec-Puka A, Ciechomska I, Morga J, Matusiak A (1999) Contents of myosin heavy chains in denervated slow and fast rat leg muscles. Comp Biochem Physiol B Biochem Mol Biol 122 (3):355-362

Jakubiec-Puka A, Kordowska J, Catani C, Carraro U (1990) Myosin heavy chain isoform composition in striated muscle after denervation and self-re-innervation. Eur J Biochem 193 (3):623-628

Janacek J, Cebasek V, Kubinova L, Ribaric S, Erzen I (2009) 3D visualization and measurement of capillaries supplying metabolically different fiber types in the rat extensor digitorum longus muscle during denervation and re-innervation. $\mathrm{J}$ Histochem Cytochem 57 (5):437-447. doi:10.1369/jhc.2008.953018

Jaweed MM, Herbison GJ, Ditunno JF (1975) Denervation and re-innervation of fast and slow muscles. A histochemical study in rats. Journal of Histochemistry \& Cytochemistry 23 (11):808-827. doi:10.1177/23.11.127809

Kissane RWP, Wright O, Al'Joboori YD, Marczak P, Ichiyama RM, Egginton S (2018) Effects of treadmill training on microvascular remodeling in the rat after spinal cord injury. Muscle Nerve. doi:10.1002/mus.26379

Kugler P (1991) Microphotometric determination of enzymes in brain sections. V. Glycerophosphate dehydrogenases. Histochemistry 95 (6):579-583

Lang F, Khaghani S, Turk C, Wiederstein JL, Holper S, Piller T, Nogara L, Blaauw B, Gunther S, Muller S, Braun T, Kruger M (2018) Single Muscle Fiber Proteomics Reveals Distinct Protein Changes in Slow and Fast Fibers during Muscle Atrophy. J Proteome Res. doi:10.1021/acs.jproteome.8b00093

Large J, Tyler KR (1985) Changes in capillary distribution in rat fast muscles following nerve crush and re-innervation. J Physiol 362:13-21. 
Lexell J, Taylor C, Sjöström M (1985) Analysis of sampling errors in biopsy techniques using data from whole muscle cross sections. J Appl Physiol 59 (4):1228-1235

Liu L, Xie F, Wei K, Hao XC, Li P, Cao J, Min S (2016) Sepsis induced denervation-like changes at the neuromuscular junction. The Journal of surgical research 200 (2):523-532. doi:10.1016/j.jss.2015.09.012

Lucas CA, Kang LH, Hoh JF (2000) Monospecific antibodies against the three mammalian fast limb myosin heavy chains. Biochem Biophys Res Commun 272 (1):303-308. doi:10.1006/bbrc.2000.2768

Michel R, Parry D, Dunn S (1996) Regulation of myosin heavy chain expression in adult rat hindlimb muscles during short-term paralysis: Comparison of denervation and tetrodotoxin-induced neural inactivation. FEBS Lett 391 (1-2):39-44. doi:10.1016/0014-5793(96)00618-7

Midrio M (2006) The denervated muscle: facts and hypotheses. A historical review. Eur J Appl Physiol 98 (1):1-21. doi:10.1007/s00421-006-0256-z

Nachmias VT, Padykula HA (1958) A histochemical study of normal and denervated red and white muscles of the rat. J Biophys Biochem Cytol 4 (1):47-54

Novikoff AB, Shin WY, Drucker J (1961) Mitochondrial localization of oxidative enzymes: staining results with two tetrazolium salts. J Biophys Biochem Cytol 9:47-61

Olfert IM, Baum O, Hellsten Y, Egginton S (2016) Advances and challenges in skeletal muscle angiogenesis. Am J Physiol Heart Circ Physiol 310 (3):H326-336. doi:10.1152/ajpheart.00635.2015

Olfert IM, Birot O (2011) Importance of anti-angiogenic factors in the regulation of skeletal muscle angiogenesis. Microcirculation (New York, NY : 1994) 18 (4):316-330. doi:10.1111/j.1549-8719.2011.00092.x

Otis JS, Roy RR, Edgerton VR, Talmadge RJ (2004) Adaptations in metabolic capacity of rat soleus after paralysis. J Appl Physiol 96 (2):584-596. Epub 2003 Oct 2017.

Paudyal A, Slevin M, Maas H, Degens H (2018) Time course of denervation-induced changes in gastrocnemius muscles of adult and old rats. Exp Gerontol 106:165-172. doi:10.1016/j.exger.2018.03.008

Pette D, Staron RS (1988) Molecular basis of the phenotypic characteristics of mammalian muscle fibres. Ciba Found Symp 138:2234

Piasecki M, Ireland A, Coulson J, Stashuk DW, Hamilton-Wright A, Swiecicka A, Rutter MK, McPhee JS, Jones DA (2016) Motor unit number estimates and neuromuscular transmission in the tibialis anterior of master athletes: evidence that athletic older people are not spared from age-related motor unit remodeling. Physiol Rep 4 (19). doi:10.14814/phy2.12987

Planitzer G, Miethke A, Baum O (2001) Nitric oxide synthase-1 is enriched in fast-twitch oxidative myofibers. Cell Tissue Res 306 (2):325-333. doi:10.1007/s004410100449

Reichmann H, Pette D (1984) Glycerolphosphate oxidase and succinate dehydrogenase activities in IIA and IIB fibres of mouse and rabbit tibialis anterior muscles. Histochemistry 80 (5):429-433

Ribarič S, Čebašek V (2013) Simultaneous visualization of myosin heavy chain isoforms in single muscle sections. Cells Tissues Organs 197 (4):312-321. doi:10.1159/000345424

Romanul FC, Hogan EL (1965) Enzymatic changes in denervated muscle. I. Histochemical studies. Arch Neurol 13 (3):263-273

Roy RR, Zhong H, Hodgson JA, Grossman EJ, Siengthai B, Talmadge RJ, Edgerton VR (2002) Influences of electromechanical events in defining skeletal muscle properties. Muscle Nerve 26 (2):238-251.

Schiaffino S, Dyar KA, Ciciliot S, Blaauw B, Sandri M (2013) Mechanisms regulating skeletal muscle growth and atrophy. FEBS J 280 (17):4294-4314. doi:10.1111/febs.12253

Schiaffino S, Gorza L, Sartore S, Saggin L, Ausoni S, Vianello M, Gundersen K, Lømo T (1989) Three myosin heavy chain isoforms in type 2 skeletal muscle fibres. J Muscle Res Cell Motil 10 (3):197-205

Schiaffino S, Reggiani C (1994) Myosin isoforms in mammalian skeletal muscle. J Appl Physiol 77 (2):493-501

Schiaffino S, Reggiani C (1996) Molecular diversity of myofibrillar proteins: gene regulation and functional significance. Physiol Rev 76 (2):371-423

Schiaffino S, Reggiani C (2011) Fiber types in mammalian skeletal muscles. Physiol Rev 91 (4):1447-1531. doi:10.1152/physrev.00031.2010

Simon M, Porter R, Brown R, Coulton GR, Terenghi G (2003) Effect of NT-4 and BDNF delivery to damaged sciatic nerves on phenotypic recovery of fast and slow muscles fibres. The European journal of neuroscience 18 (9):2460-2466

Staron RS, Pette D (1993) The continuum of pure and hybrid myosin heavy chain-based fibre types in rat skeletal muscle. Histochemistry 100 (2):149-153

Sun H, Liu J, Ding F, Wang X, Liu M, Gu X (2006) Investigation of differentially expressed proteins in rat gastrocnemius muscle during denervation-re-innervation. Journal of muscle research and cell motility 27 (3-4):241-250. doi:10.1007/s10974-0069067-4

Tomanek RJ, Lund DD (1973) Degeneration of different types of skeletal muscle fibres. I. Denervation. J Anat 116 (Pt 3):395-407

Tyml K, Mathieu-Costello O, Cheng L, Noble EG (1999) Differential microvascular response to disuse in rat hindlimb skeletal muscles. J Appl Physiol (1985) 87 (4):1496-1505

Vacca LL (1985) Laboratory manual of histochemistry Raven Press, New York

Wagatsuma A, Tamaki H, Ogita F (2005) Capillary supply and gene expression of angiogenesis-related factors in murine skeletal muscle following denervation. Exp Physiol 90 (3):403-409. Epub 2005 Feb 2011.

Waters RE, Rotevatn S, Li P, Annex BH, Yan Z (2004) Voluntary running induces fiber type-specific angiogenesis in mouse skeletal muscle. Am J Physiol Cell Physiol 287 (5):C1342-C1348. doi:10.1152/ajpcell.00247.2004

Wu P, Chawla A, Spinner RJ, Yu C, Yaszemski MJ, Windebank AJ, Wang H (2014) Key changes in denervated muscles and their impact on regeneration and re-innervation. Neural Regen Res 9 (20):1796-1809. doi:10.4103/1673-5374.143424 


\section{Figures legends}

Fig.1 Fiber type composition and capillary density in rat extensor digitorum muscle (EDL) from (a) control at the start of the experiment $\mathrm{CON}-1,(\mathbf{b})$ control at the end of the experiment $\mathrm{CON}-2$, (c) permanent denervation (NC group) at 4 weeks after nerve cut and (d) transient denervation /re-innervation (Double NCR group) at 4 weeks after second nerve crush. Multicolor fluorescence simultaneously demonstrates the three myosin heavy chains (MyHC) isoforms (type-1, $-2 \mathrm{a}$ and $-2 \mathrm{~b}$ ) and the capillaries. The MyHC isoform type-1 is labelled with primary antibody BA-D5 and visualized by (blue) Alexa Fluor - 350; the MyHC isoform type-2a is labelled with primary antibody SC-71 and visualized by (red) Alexa Fluor - 546 and the MyHC isoform type-2b is labelled with primary antibody BF-F3 and visualized by (green) Alexa Fluor - 488. The pure muscle fibers predominately express only one MyHC isoform as: blue (type-1), red (type-2a), black (type 2x) and green profiles (type-2b); and the hybrid muscle fibers express two (or more) MyHC isoforms simultaneous as: violet (type 1/2a), dark red (type 2ax) and dark green profiles (type 2xb). Double stained capillaries (with fluorescein and rhodamin labelled lectin GSL-I) are visible as yellow profiles due to colocalization of green and red fluorescence. Endomysium and perimysium are unstained. Bar equals $100 \mu \mathrm{m}$.

Fig.2 Fiber type differentiations as revealed by dark microscopy of a multiple-stained single tissue cross section (a-d) and by light microscopy of single-stained tissue cross sections (in series) (e-1) of rat EDL from the start of the experiment (CON-1 group).

(a) Merged view of a multicolor fluorescence (RGB image) where different pure and hybrid MyHC muscle fiber types and capillaries are shown simultaneously and greyscale single channel views (of the same RGB image), where each of the MyHC isoform type is shown (in grey fibers) in a separate channel; (b) in the blue channel is the MyHC isoform type-1; (c) in the red channel is the MyHC isoform type-2a and (d) in the green channel is the MyHC type-2b isoform. Views of single-stained tissue sections, where each MyHC isoform is visualized by an immunoperoxidase reaction (in brown fibers) in a separate tissue section; (e) MyHC isoform type-2x (labelled by antibody 6H1); (f) MyHC isoform type-1 (labelled by antibody BA-D5); (g) MyHC isoform type-2a (labelled by antibody Sc-71) and (h) MyHC isoform type-2b (labelled by antibody BF-F3). Note the likeness between the immune-fluorescent (left column) and the immune-peroxidase staining (middle column) for: muscle fibers type-1 (b, f), type-2a (c, g), and type-2b (d, h). (i) MyHC type-2x muscle fibers are shown as light (unstained) fibers, since antibody BF-35 labelled all MyHC isoforms except of type-2x; (j) a general view of histo-pathology shown by hematoxylin and eosin reaction, the histochemical activity of (k) succinate dehydrogenase (SDH) (l) and $\alpha$-glycerophosphate dehydrogenase ( $\alpha$-GPDH) enzymes. Red arrow designates the same muscle fiber (the type-2x) throughout the series. Bar equals $100 \mu \mathrm{m}$.

Fig.3 Fiber type differentiations as revealed by dark microscopy (a-d) of a multiple-stained single tissue cross section and by light microscopy (e-1) of single-stained tissue cross sections (in series) of rat EDL from the end of the experiment CON-2 group. 
(a) Merged view of a multicolor fluorescence (RGB image) where different pure and hybrid MyHC muscle fiber types and capillaries are shown simultaneously and greyscale single channel views (of the same RGB image), where each of the MyHC isoform type is shown (in grey fibers) in a separate channel; (b) in the blue channel is the MyHC isoform type-1; (c) in the red channel is the MyHC isoform type-2a and (d) in the green channel is the MyHC type-2b isoform. Views of single-stained tissue sections, where each MyHC isoform is visualized by an immunoperoxidase reaction (in brown fibers) in a separate tissue section; (e) MyHC isoform type-2x (labelled by antibody 6H1); (f) MyHC isoform type-1 (labelled by antibody BA-D5); (g) MyHC isoform type-2a (labelled by antibody Sc-71) and (h) MyHC isoform type-2b (labelled by antibody BF-F3). Note the likeness between the immune-fluorescent (left column) and the immune-peroxidase staining (middle column) for: muscle fibers type-1 (b, f), type-2a (c, g), and type-2b (d, h). (i) MyHC type-2x muscle fibers are shown as light (unstained) fibers, since antibody BF-35 labelled all MyHC isoforms except of type-2x; (j) a general view of the histo-pathology shown by hematoxylin and eosin reaction, the histochemical activity of (k) succinate dehydrogenase (SDH) and (l) $\alpha$-glycerophosphate dehydrogenase ( $\alpha$-GPDH) enzymes. Red arrow designates the same muscle fiber (of type-2x) throughout the series. Bar equals $100 \mu \mathrm{m}$.

Fig.4 Fiber type differentiations as revealed by dark microscopy (a-d) of a multiple-stained single tissue cross section and by light microscopy (e-l) of single-stained tissue cross sections (in series) of rat EDL 4 weeks after nerve cut in permanent denervation (NC group).

(a) Merged view of a multicolor fluorescence (RGB image) where different pure and hybrid MyHC muscle fiber types and capillaries are shown simultaneously and greyscale single channel views (of the same RGB image), where each of the MyHC isoform type is shown (in grey fibers) in a separate channel (b) in the blue channel is the MyHC isoform type-1; (c) in the red channel is the MyHC isoform type-2a and (d) in the green channel is the MyHC type-2b isoform. Views of single-stained tissue sections, where each MyHC isoform is visualized by an immunoperoxidase reaction (in brown fibers) in a separate tissue section; (e) MyHC isoform type-2x (labelled by antibody 6H1); (f) MyHC isoform type-1 (labelled by antibody BA-D5); (g) MyHC isoform type-2a (labelled by antibody Sc-71) and (h) MyHC isoform type-2b (labelled by antibody BF-F3). Note the likeness between the immune-fluorescent (left column) and the immune-peroxidase staining (middle column) for: muscle fibers type-1 (b, f), type-2a (c, g), and type-2b (d, h). (i) MyHC type-2x muscle fibers are shown as light (unstained) fibers, since antibody BF-35 labelled all MyHC isoforms except of type-2x; (j) a general view of histo-pathology shown by hematoxylin and eosin reaction, the histochemical activity of (k) succinate dehydrogenase (SDH) and (l) $\alpha$-glycerophosphate dehydrogenase ( $\alpha$-GPDH) enzymes. Red arrow designates the same (type-2x) muscle fiber throughout the series. Bar equals $100 \mu \mathrm{m}$. 
Fig.5 Fiber type differentiations as revealed by dark microscopy (a-d) of a multiple-stained single tissue cross section and by light microscopy (e-l) of single-stained tissue cross sections (in series) of rat EDL 4 weeks after second nerve crush in transient denervation group (Double NCR group).

(a) Merged view of a multicolor fluorescence (RGB image) where different pure and hybrid MyHC muscle fiber types and capillaries are shown simultaneously (details in Materials and methods) and greyscale single channel views (of the same RGB image), where each MyHC isoform type is shown (in grey fibers) in a separate channel (b) in a single blue channel the MyHC isoform type-1 (c) in a single red channel the MyHC isoform type-2a and (d) in a green channel the MyHC type-2b isoform. Views of single-stained tissue sections, where each MyHC isoform is visualized by an immunoperoxidase reaction (in brown fibers) in a separate tissue section; (e) MyHC isoform type-2x (labelled by antibody 6H1); (f) MyHC isoform type-1 (labelled by antibody BA-D5); (g) MyHC isoform type-2a (labelled by antibody Sc-71) and (h) MyHC isoform type-2b (labelled by antibody BF-F3). Note the likeness between the immune-fluorescent (left column) and the immune-peroxidase staining (middle column) for: muscle fibers type-1 (b, f), type-2a (c, g), and type-2b (d, h). (i) MyHC type-2x muscle fibers are shown as light (unstained) fibers, since antibody BF-35 labelled all MyHC isoforms except of type-2x, (j) general view of histo-pathology shown by hematoxylin and eosin reaction, the histochemical activity of (k) succinate dehydrogenase (SDH) and (l) $\alpha$-glycerophosphate dehydrogenase ( $\alpha$-GPDH) enzymes. Red arrow designates the same muscle fiber (of type-2x) throughout the series. Bar equals $100 \mu \mathrm{m}$.

Fig. 6 Comparison of numerical $(\mathrm{N} \%)$ and areal (A\%) proportions in the rat EDL muscles following nerve injury between five groups of MyHC fibers types - of four individual pure fiber types (-1, -2a, -2x,-2b) and of pooled hybrid muscle fiber types. The numbers of fibers included in each of the MyHC fiber type groups are: 73 for type-1, 811 for type-2a, 1252 for type-2x, 1110 for type-2b and 865 for a group of pooled hybrid fiber types. The number of animals per group was 4. Abbreviations: CON-1 (C1), the control group at the start of the experiment; CON-2 (C2), the control group at the end of the experiment; NC (D), the permanent denervation group at 4 weeks after nerve cut; Double NCR (R), the transient denervation group at 4 weeks after second nerve crush.

Statistical differences between groups for N\%: type-2b R < C1, C2, D ( p <0.001) and pooled hybrid fibers types R > C1, C2, D (p<0.01); for A\%: type-2a D > C1, C2 (p<0.05), type-2b D < C1, C2 (p<0.01), type-2x R > C1, C2, D (p<0.01), type-2b R < C1, $\mathrm{C} 2, \mathrm{D}(\mathrm{p}<0.001)$ and pooled hybrid fibers types $\mathrm{R}>\mathrm{C} 1, \mathrm{C} 2,(\mathrm{p}<0.01), \mathrm{R}>\mathrm{D}(\mathrm{p}<0.05)$.

Fig. 7 Histochemical activity of oxidative metabolic enzymes succinate dehydrogenase (SDH) and NADH tetrazolium reductase (TR) and the glycolytic enzyme $\alpha$-glycerophosphate dehydrogenase ( $\alpha$-GPDH) in rat EDL muscle of CON-1, control group at the 
start of the experiment; $\mathrm{CON}-2$, control group at the end of the experiment; $\mathrm{NC}$, permanent denervation group at 4 weeks after nerve cut; Double NCR, transient denervation group at 4 weeks after second nerve crush. Bar $100 \mu \mathrm{m}$.

Fig. 8 Number of capillaries around a fiber $(\mathrm{CAF})$ and CAF per fiber perimeter $\left(\mathrm{CAF} / \mathrm{FP}\left(\mu \mathrm{m}^{-1}\right)\right)$ of four pure $(-1,-2 \mathrm{a}, 2 \mathrm{x}$ and $2 \mathrm{~b})$ and two most frequent hybrid MyHC muscle fibers types (-2ax and-2xb) in rat EDL muscles of CON-1 (C1), control group from the start of the experiment; CON-2 (C2), control group from the end of the experiment; NC (D), permanent denervation group from 4 weeks after nerve cut; Double NCR (R), transient denervation group from 4 weeks after second nerve crush. P values: * $p<$ $0.05, * * p<0.01 ; * * * p<0.001$. Values are means $\pm \mathrm{SD}$. 


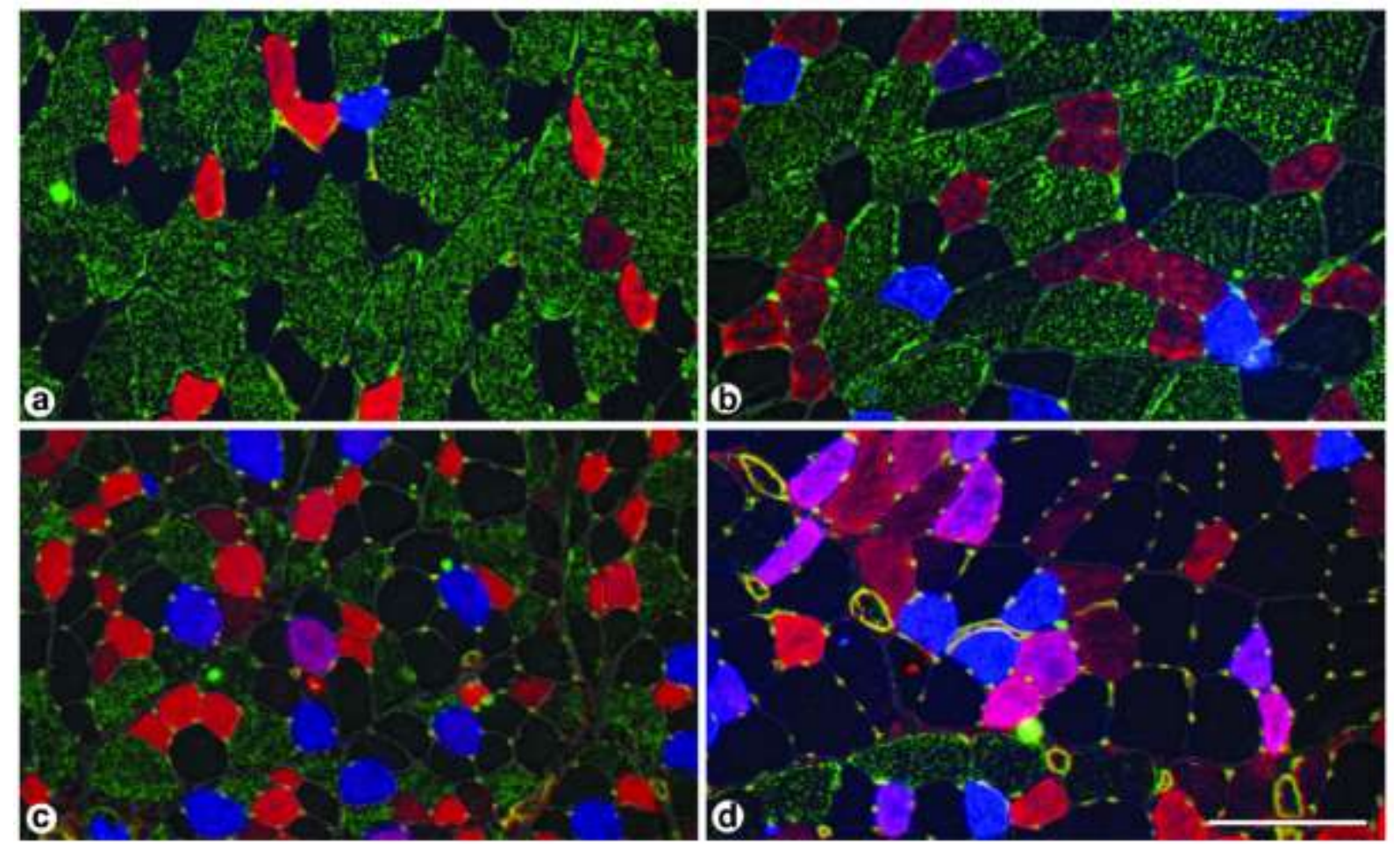



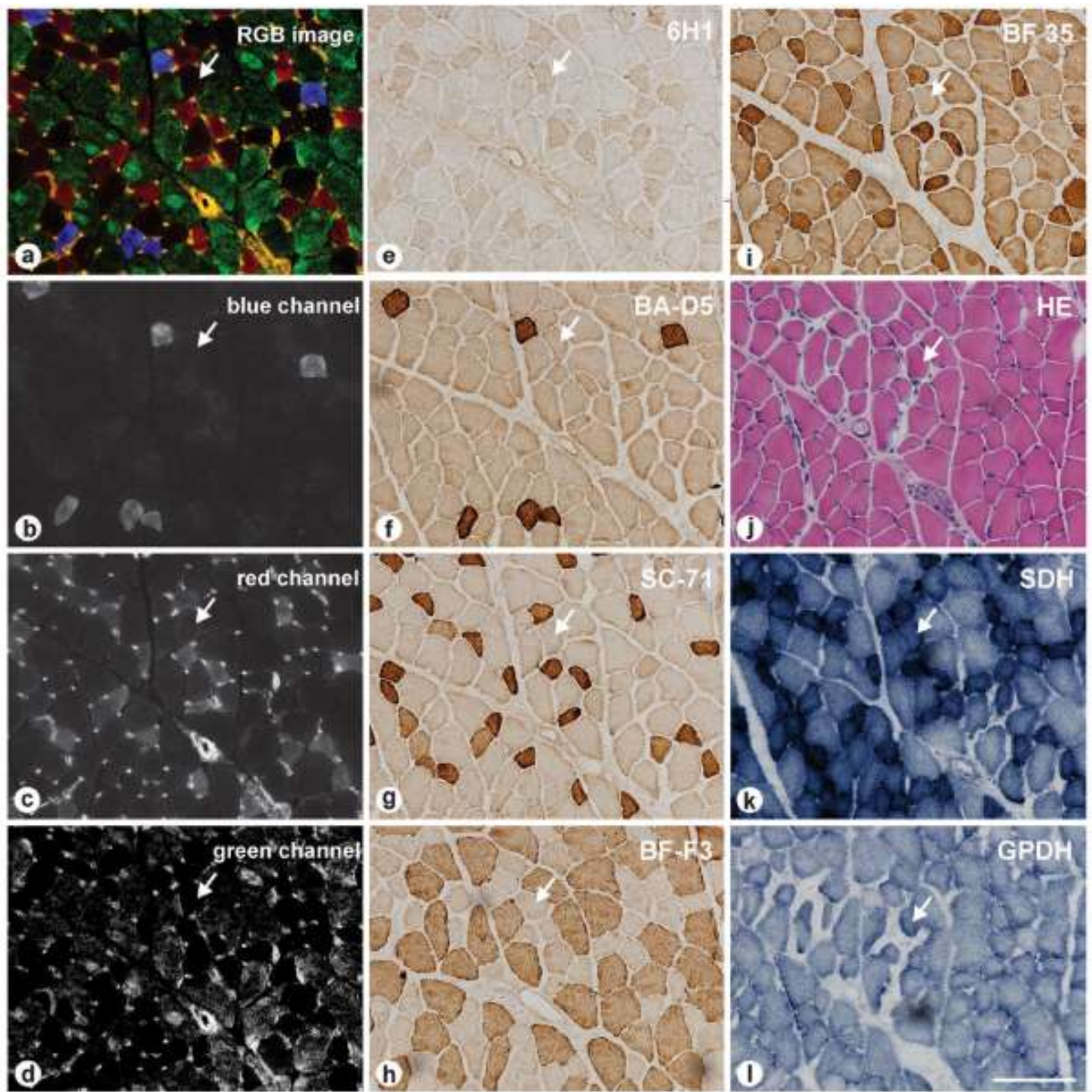


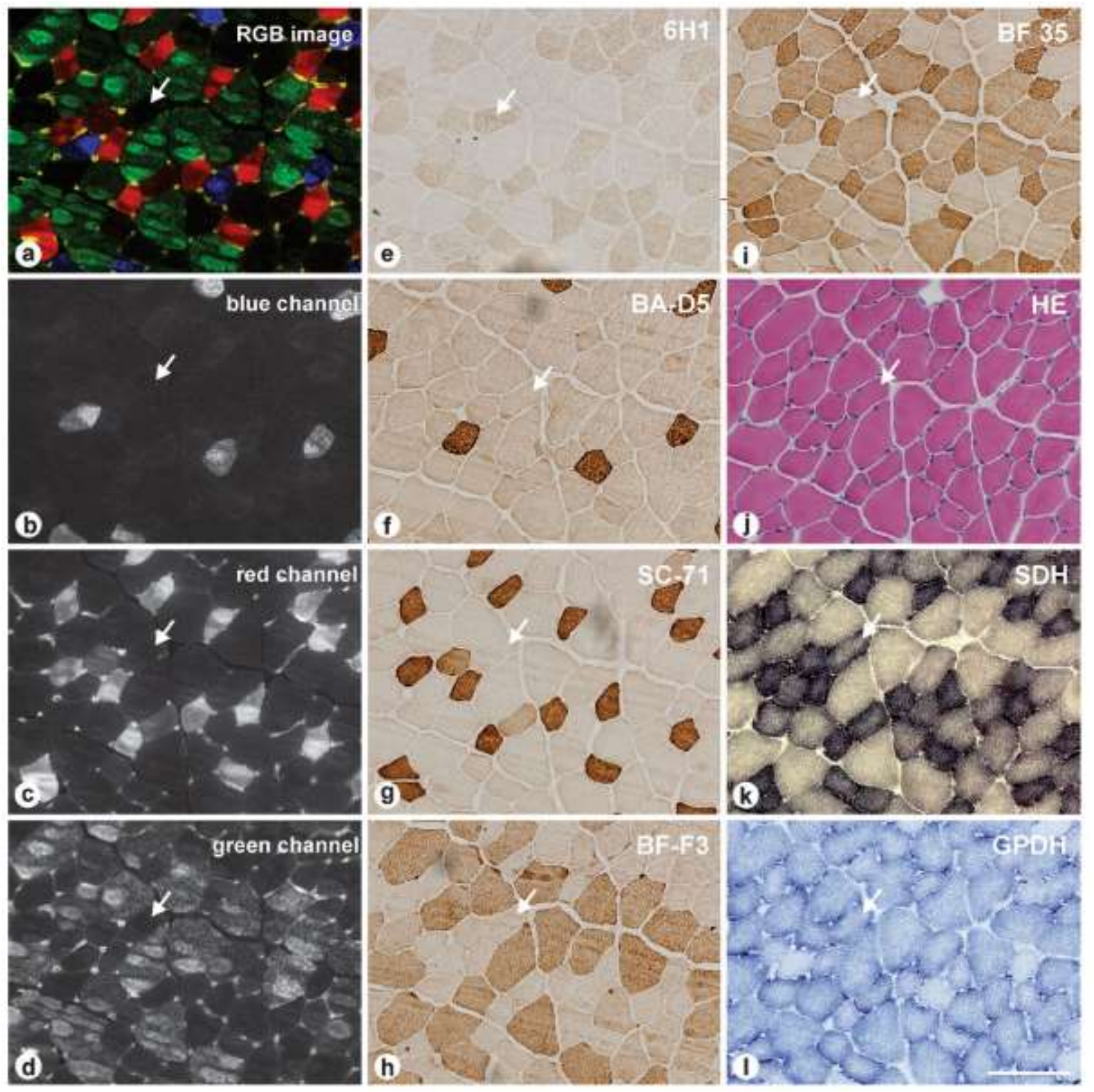




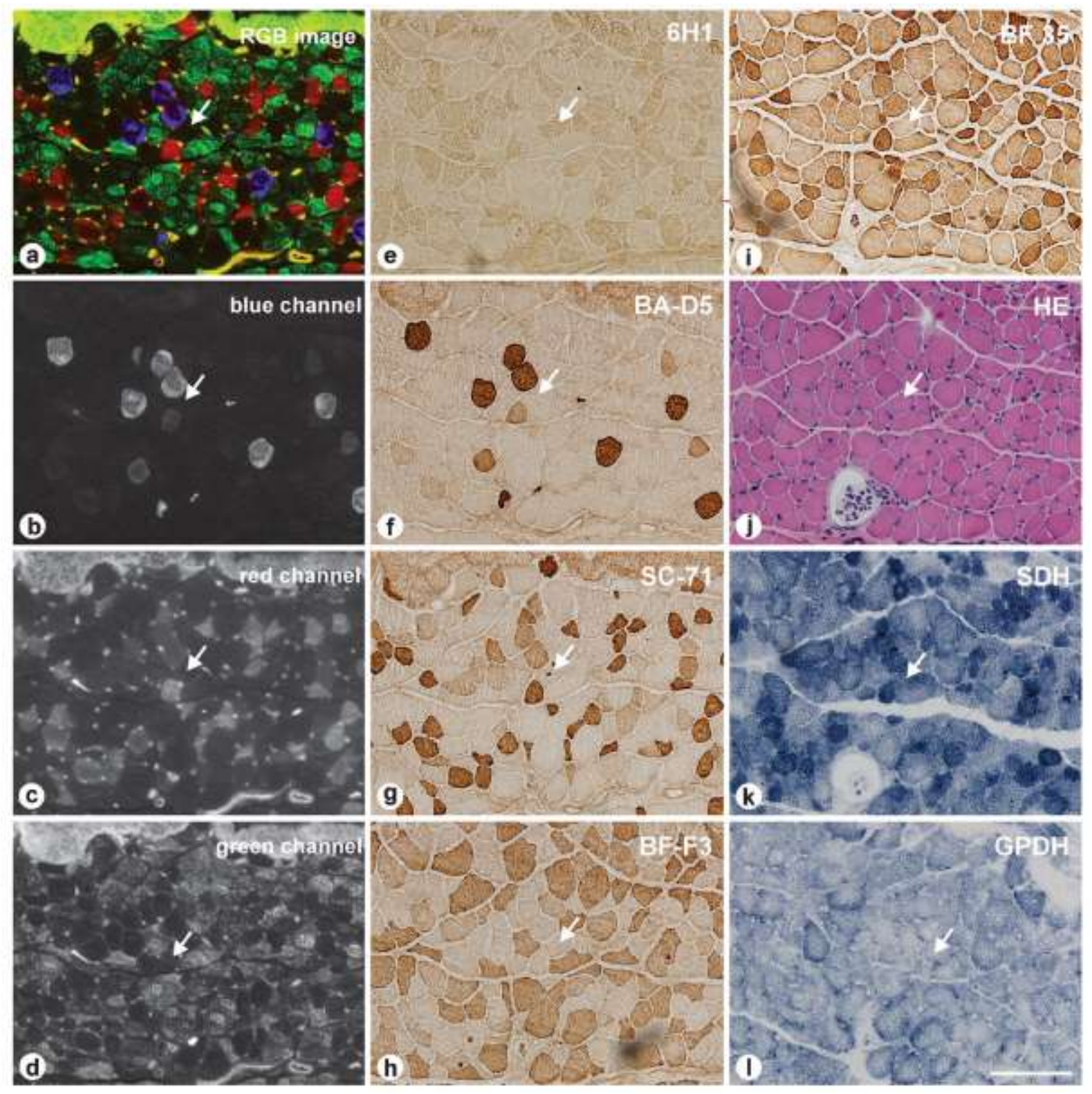



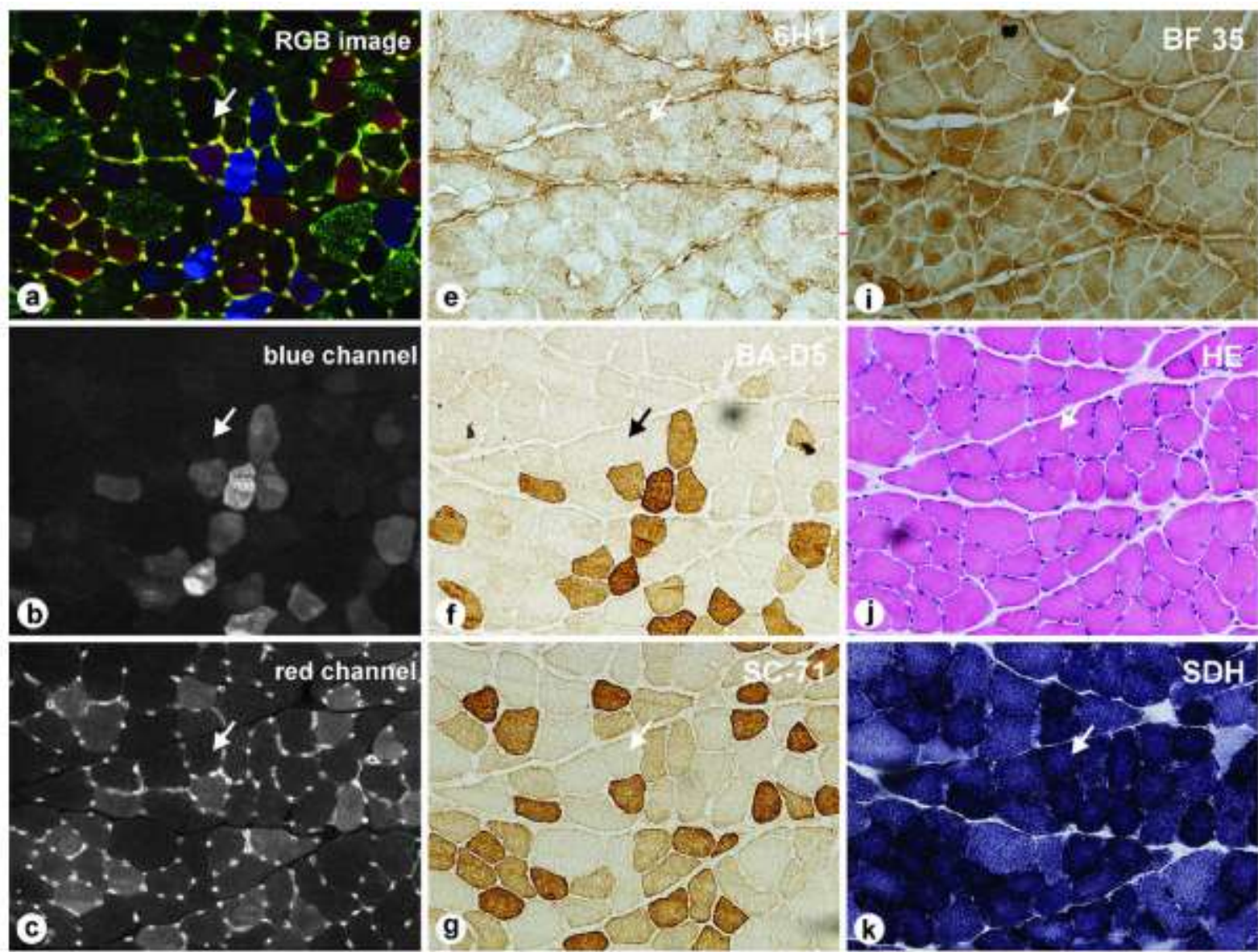

c.
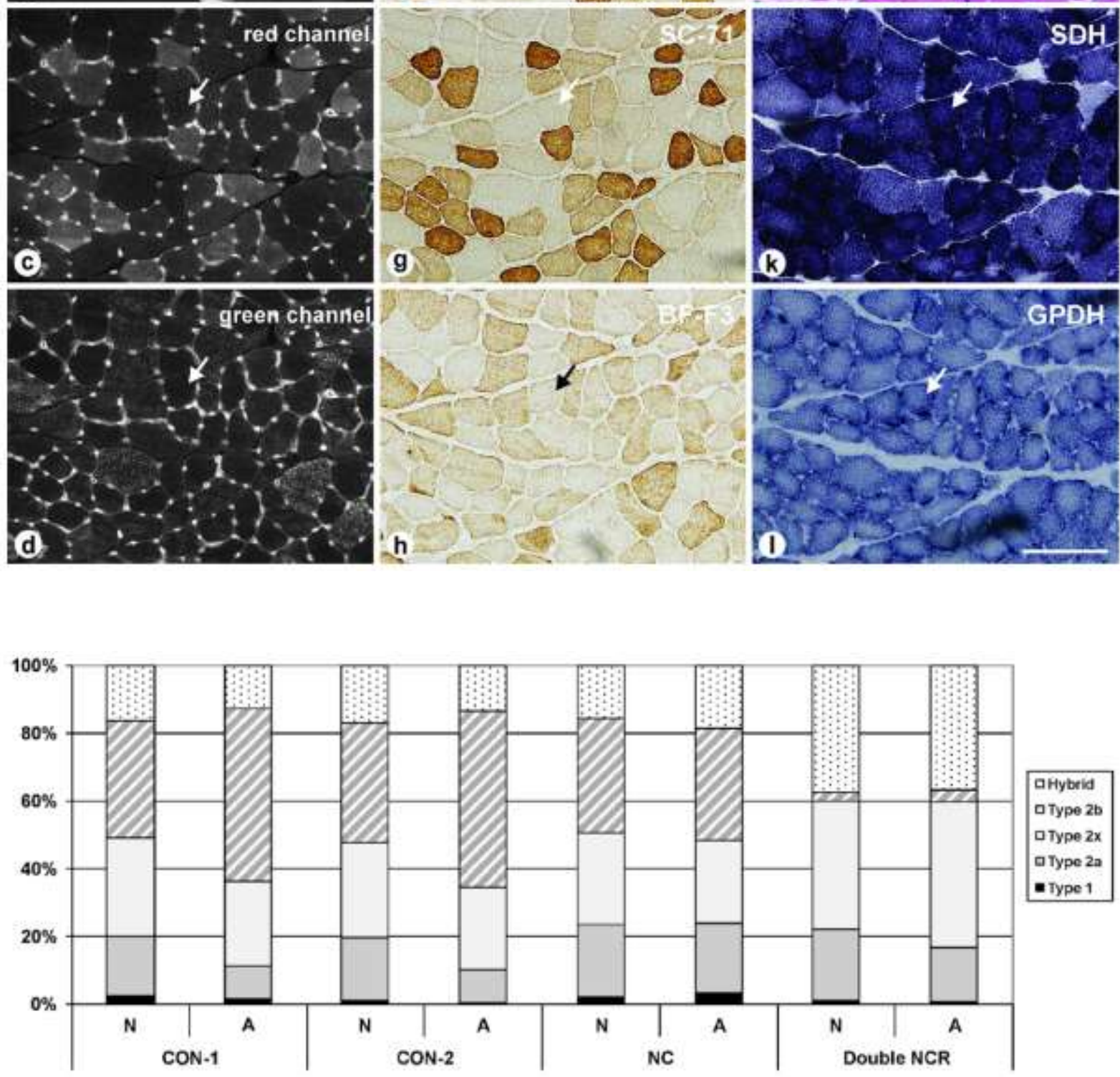


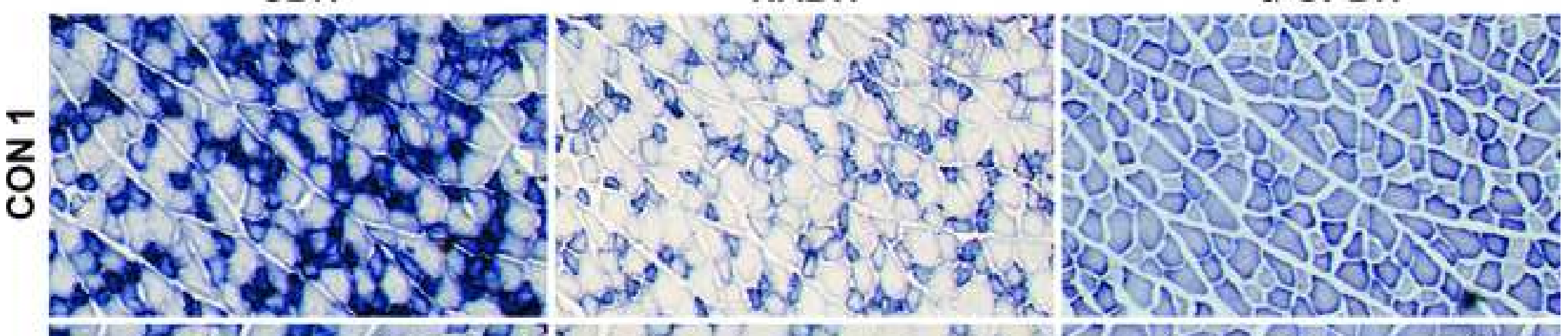

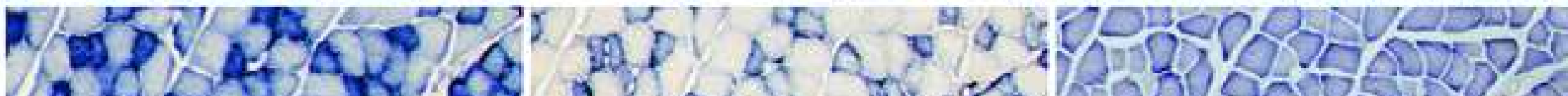

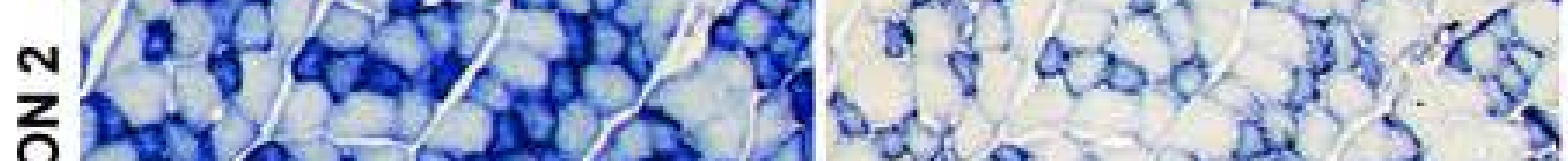

ठํ.

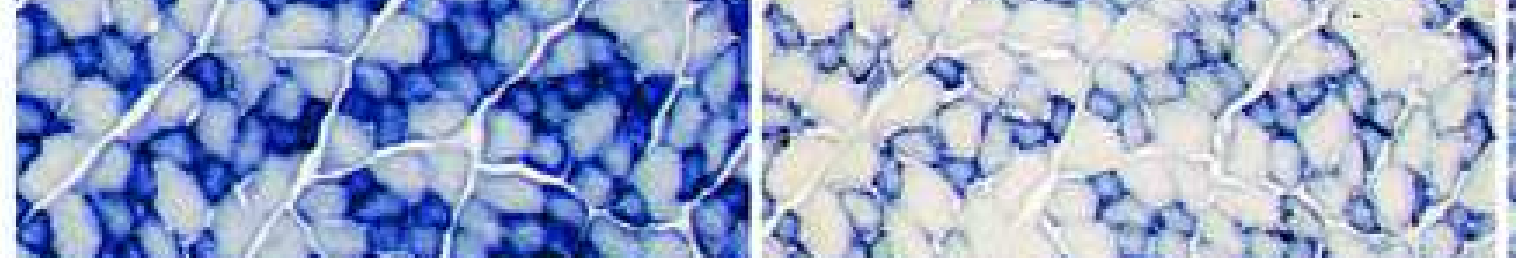
0000000

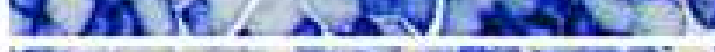
7.2.

u

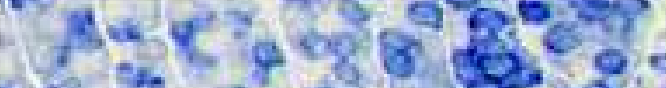

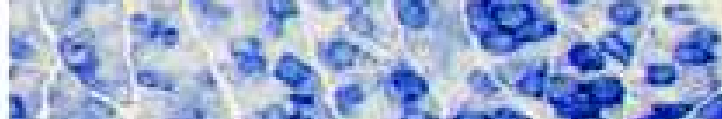

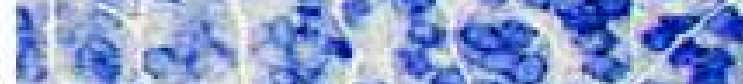

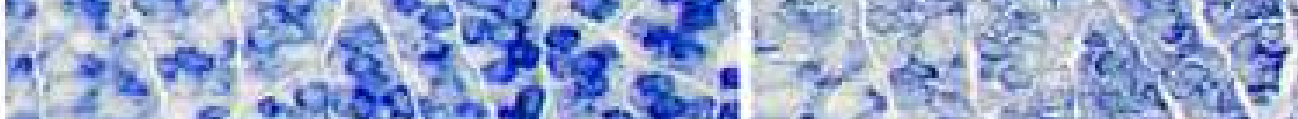

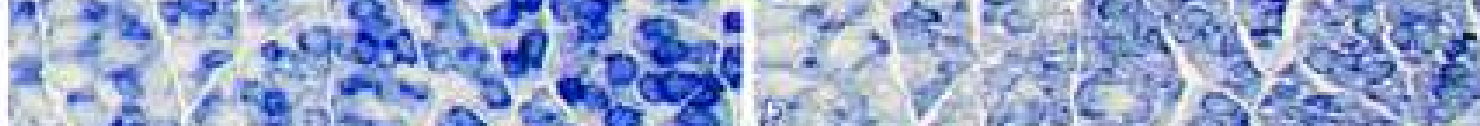

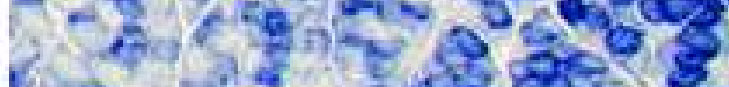

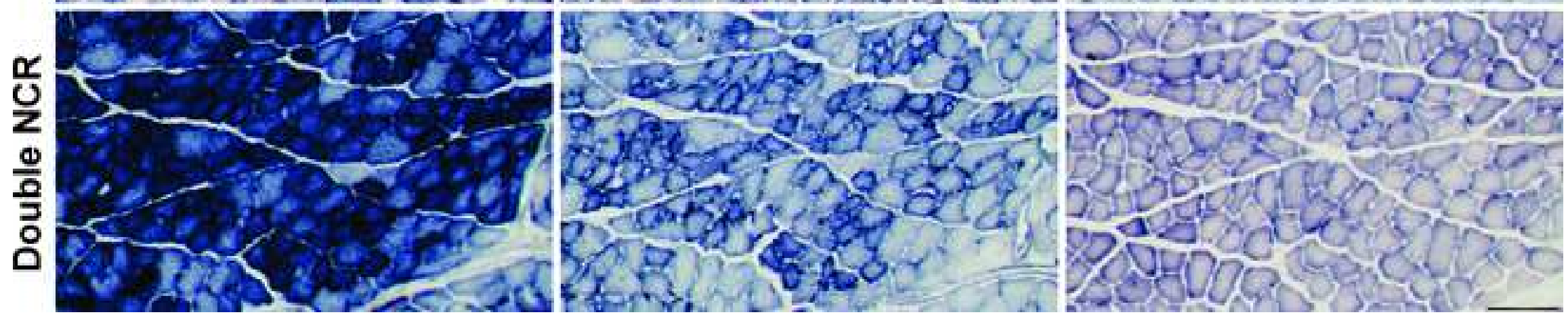



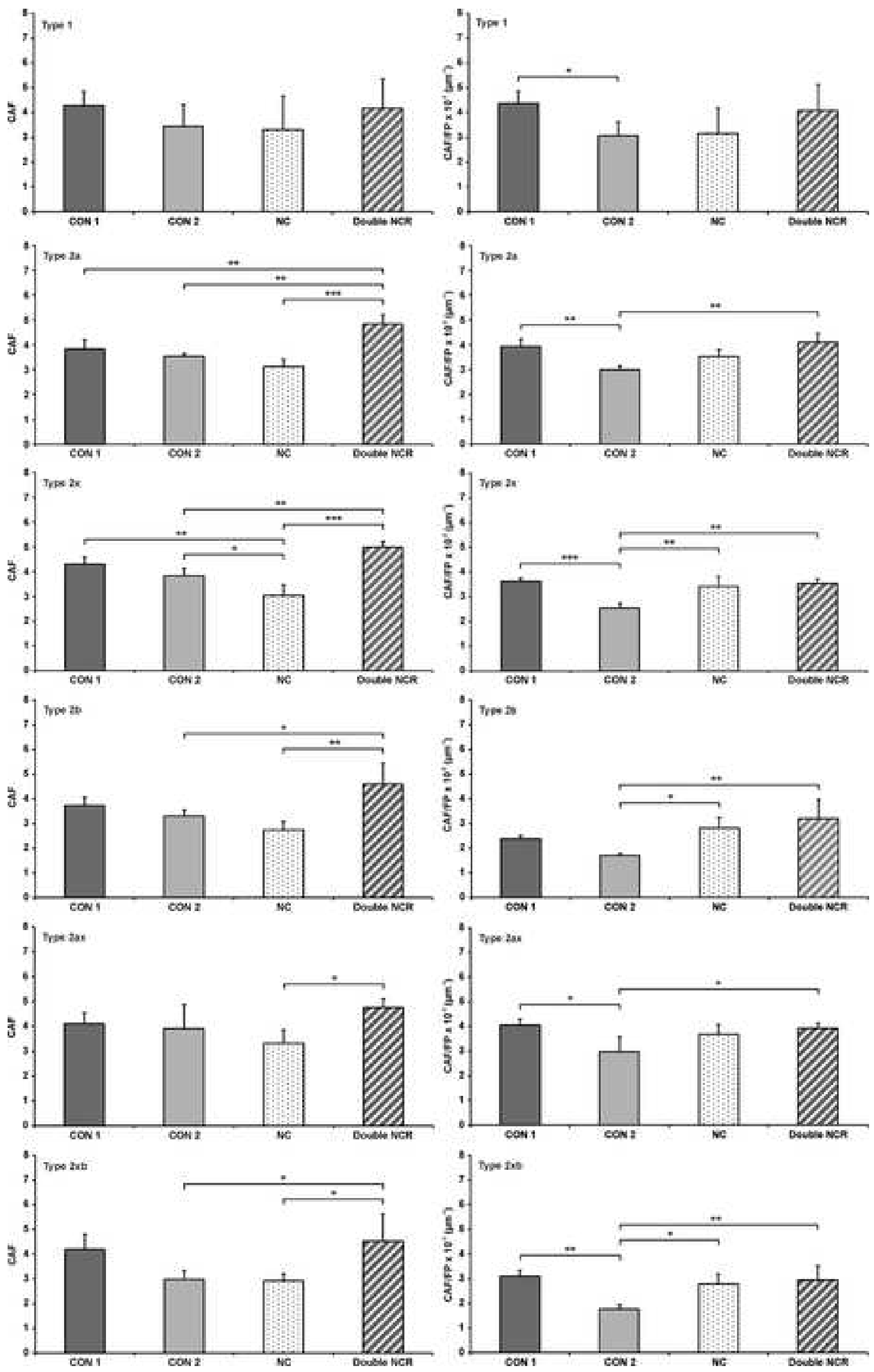


\section{Tables Headings}

Table 1 Experimental protocol. Animals were randomly divided into four groups (4 animals per group). In the CON-1 group, the left EDL muscle was isolated on day 0 - at the start of the experiment and in the CON-2 group on day 42 - at the end of the experiment. In the NC group (permanent denervation group) the left sciatic nerve was cut on day 14 and the left EDL was isolated on day 42 - 4 weeks after nerve cut. In the Double NCR group (transient denervation group), the first crush of the left sciatic nerve was done on day 0, the second crush on day 14, and the left EDL was isolated on day 42.

Table 2 Experimental numbers of muscle fibers. The numbers of muscle fibers analyzed in the rat EDL muscle are per each MyHC fiber type and per each animal group. Note that among the pure MyHC fiber types the least frequent were fibers of type-1 and among hybrid MyHC fiber types the most frequent were fibers of type-2ax and of type-2xb.

Table 3 Comparisons of MyHC fibers types perimeters (FP, units in $\mu \mathrm{m}$ ) in the rat EDL between and within groups. Note that in NC group differences between different MyHC fibers types disappeared. 
Table 1

\begin{tabular}{|l|l|l|l|}
\hline \multirow{2}{*}{ Animal groups } & \multicolumn{3}{|c|}{ Time } \\
\cline { 2 - 4 } & Day 0 & Day 14 & Day 42 \\
\hline CON-1 & Isolation of left EDL & \multicolumn{1}{|c|}{$/$} \\
\hline CON-2 & \multicolumn{1}{|c|}{$/$} & \multicolumn{1}{|c|}{$/$} & Isolation of left EDL \\
\hline NC & $\begin{array}{l}\text { Left sciatic nerve cut } \\
\text { crush }\end{array}$ & Isolation of left EDL \\
\hline Double NCR & $\begin{array}{l}\text { 2. left sciatic nerve } \\
\text { crush }\end{array}$ & Isolation of left EDL \\
\hline
\end{tabular}

Title: Experimental protocol

CON-1, control group at the start of the experiment;

CON-2, control group at the end of the experiment;

NC complete denervation group from 4 weeks after nerve cut;

Double NCR, transient denervation group from 4 weeks after double nerve crush. Four animals were in each group.

Table 2

\begin{tabular}{|c|c|c|c|c|c|c|c|c|c|c|c|c|}
\hline \multirow[b]{2}{*}{ Group } & \multirow[b]{2}{*}{$\mathrm{N}$} & \multicolumn{10}{|c|}{ MyHC fiber type (n) } & \multirow[b]{2}{*}{ Sum } \\
\hline & & 1 & $2 \mathrm{a}$ & $2 \mathrm{x}$ & $2 \mathrm{~b}$ & $1 / 2 \mathrm{a}$ & $1 / 2 \mathrm{axb}$ & $* 1 / 2 \mathrm{x}$ & $2 \mathrm{ax}$ & $2 a x b$ & $2 \mathrm{xb}$ & \\
\hline$\overline{\mathrm{CON}-1}$ & 4 & 24 & 157 & 263 & 315 & 8 & 1 & 16 & 70 & 1 & 50 & 904 \\
\hline $\mathrm{CON}-2$ & 4 & 14 & 216 & 329 & 409 & - & - & 37 & 85 & - & 77 & 1168 \\
\hline $\mathrm{NC}$ & 4 & 22 & 224 & 276 & 349 & 9 & 1 & 1 & 61 & - & 86 & 1029 \\
\hline Double NCR & 4 & 13 & 214 & 384 & 27 & 26 & - & 4 & 196 & 24 & 129 & 1017 \\
\hline & $\mathrm{um}$ & 73 & 811 & 1252 & 1100 & 43 & 2 & 58 & 412 & 25 & 342 & 4118 \\
\hline
\end{tabular}

Title: Experimental numbers of muscle fibers per fiber type.

CON-1, control group from the start of the experiment;

CON-2, control group from the end of the experiment;

$\mathrm{NC}$, group from 4 weeks after complete denervation - after nerve cut;

Double NCR, group from 4 weeks after transient denervation - after double nerve crush.

* Identified by additional checking on classically stained tissue section. $\mathrm{N}=$ number of animals; $\mathrm{n}=$ number of muscle fibers. 
Table 3

\begin{tabular}{|c|c|c|c|c|c|}
\hline \multicolumn{6}{|c|}{ Fiber perimeter $(\mathrm{FP})(\mu \mathrm{m})$} \\
\hline $\begin{array}{l}\text { Fiber } \\
\text { type }\end{array}$ & $\begin{array}{c}\text { CON } 1(\mathrm{C} 1) \\
\mathrm{N}=4\end{array}$ & $\begin{array}{c}\mathrm{CON} 2(\mathrm{C} 2) \\
\mathrm{N}=4\end{array}$ & $\begin{array}{c}\text { Nerve cut NC (D) } \\
\mathrm{N}=4\end{array}$ & $\begin{array}{l}\text { Double NCR (R) } \\
\mathrm{N}=4\end{array}$ & ${ }^{\prime} p<0.05 ;{ }^{\prime} p<0.01 ; * p<0.001$ \\
\hline type 1 & $97.46 \pm 5.36$ & $110.57 \pm 13.31$ & $102.40 \pm 8.89$ & $101.23 \pm 8.99$ & n.s. \\
\hline type $2 \mathrm{a}$ & $97.18 \pm 3.62$ & $117.38 \pm 3.50^{\mathrm{a}}$ & $87.79 \pm 2.36^{b}$ & $117.67 \pm 1.97^{\mathrm{c}}$ & ${ }^{\mathrm{a}} \mathrm{C} 2>\mathrm{C} 1 *$; ${ }^{\mathrm{b}} \mathrm{D}<\mathrm{C} 1{ }^{\prime} \mathrm{C} 2{ }^{\prime} ;{ }^{\mathrm{c}} \mathrm{R}>\mathrm{C} 1 * \mathrm{D}^{*}$ \\
\hline type $2 \mathrm{x}$ & $118.52 \pm 6.99$ & $150.49 \pm 1.59^{\mathrm{a}}$ & $88.95 \pm 6.55^{\mathrm{b}}$ & $140.84 \pm 4.51^{\mathrm{c}}$ & ${ }^{\mathrm{a}} \mathrm{C} 2>\mathrm{C} 1 * ;{ }^{\circ} \mathrm{D}<\mathrm{C} 1 * \mathrm{C} 22^{*} ;{ }^{c} \mathrm{R}>\mathrm{C} 1 * \mathrm{D}^{*}$ \\
\hline type $2 b$ & $155.55 \pm 8.28$ & $194.77 \pm 9.81^{\mathrm{a}}$ & $98.04 \pm 10.76^{b}$ & $147.30 \pm 27.33^{\mathrm{c}}$ & ${ }^{\mathrm{a}} \mathrm{C} 2>\mathrm{C} 1$ '; ${ }^{\mathrm{b}} \mathrm{D}<\mathrm{C} 1{ }^{\prime \prime} \mathrm{C} 2 * ;{ }^{\mathrm{c}} \mathrm{R}>\mathrm{D} ; \mathrm{R}<\mathrm{C} 2 "$ \\
\hline tip $1 / 2 \mathrm{a}$ & $93.81 \pm 12.84$ & & $88.43 \pm 14.47^{\mathrm{a}}$ & $113.53 \pm 4.81$ & $\mathrm{D}<\mathrm{R}^{*}$ \\
\hline tip $1 / 2 x$ & $97.01 \pm 6.02$ & $110.10 \pm 10.91$ & & $106.04 \pm 10.42$ & n.s. \\
\hline type $2 a x$ & $101.78 \pm 6.12$ & $131.01 \pm 6.75^{\mathrm{a}}$ & $90.68 \pm 4.55^{b}$ & $121.80 \pm 5.64^{\mathrm{c}}$ & ${ }^{\mathrm{a}} \mathrm{C} 2>\mathrm{C} 1 *$; ${ }^{\mathrm{b}} \mathrm{D}<\mathrm{C} 2 * ;{ }^{\mathrm{c}} \mathrm{R}>\mathrm{C} 1$ ” $\mathrm{D}^{*}$ \\
\hline \multirow[t]{8}{*}{ type $2 x b$} & $134.81 \pm 8.71$ & $169.28 \pm 11.56^{\mathrm{a}}$ & $105.79 \pm 9.84^{b}$ & $152.36 \pm 10.90^{c}$ & ${ }^{\mathrm{a}} \mathrm{C} 2>\mathrm{C} 1$ "; ${ }^{\mathrm{b}} \mathrm{D}<\mathrm{C} 1{ }^{\prime} \mathrm{C} 2 *$; ${ }^{\mathrm{c}} \mathrm{R}>\mathrm{D} *$ \\
\hline & $1<2 x " 2 b * 2 x b^{*}$ & $1<2 x^{*} 2 b^{*}$ & & & \\
\hline & $2 a<2 x " 2 b * 2 x b *$ & $1<2 a^{\prime}{ }^{\prime} 2 x^{*}$ & & $1<2 x " 2 b * 2 x b *$ & \\
\hline & $2 \mathrm{x}<2 \mathrm{~b}^{*}$ & $2 a<2 x^{\prime \prime} 2 b^{*} 2 x^{*}$ & & $2 a<2 b ' 2 x b^{\prime}$ & \\
\hline & $1 / 2 a<2 x " 2 b * 2 x b *$ & $2 \mathrm{x}<2 \mathrm{~b}^{*}$ & n.s. & $1 / 2 x<2 b^{\prime} 2 x b^{\prime \prime}$ & \\
\hline & $1 / 2 x<2 x^{\prime} 2 b^{*} 2 x b^{*}$ & $1 / 2 x<2 x^{\prime} 2 b^{*} 2 x b^{*}$ & & $2 \mathrm{ax}<2 \mathrm{xb}$ & \\
\hline & $2 a x<2 b * 2 x b *$ & $2 a x<2 b * 2 x b *$ & & $2 \mathrm{xb}>2 \mathrm{ax}$ & \\
\hline & $2 x b<2 b$ & $2 x b<2 b$ & & & \\
\hline
\end{tabular}

Title: Comparisons of fibers types perimeters in rat EDL between and within groups.

CON-1 or (C1), control group from the start of the experiment;

$\mathrm{CON}-2$ or $(\mathrm{C} 2)$ control group from the end of the experiment;

$\mathrm{NC}$ or (D) group from 4 weeks after nerve cut or complete denervation;

Double NCR or (R) group from 4 weeks after double nerve crush or transient denervation.

Statistical differences of FP between and within groups are given on the right side and beneath the columns, respectively. $\mathrm{P}$ values: $p<0.05, " p<0.01 ; * p<0.001$. Values are means $\pm \mathrm{SD}$. $\mathrm{N}=$ number of animals in each group. 